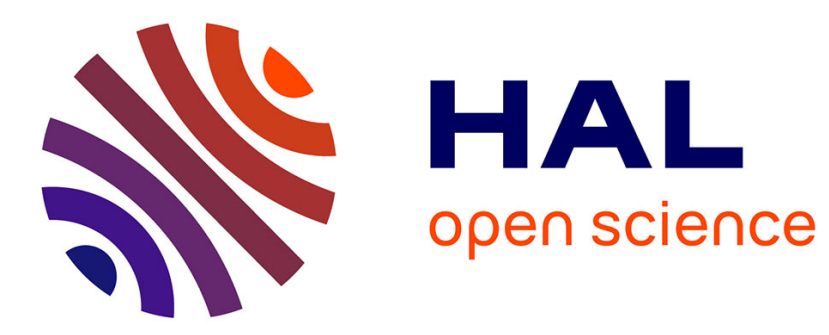

\title{
A General and Efficient Method for Solving Regime-Switching DSGE Models
}

Julien Albertini, Stéphane Moyen

\section{To cite this version:}

Julien Albertini, Stéphane Moyen. A General and Efficient Method for Solving Regime-Switching DSGE Models. 2020. halshs-03067554

\section{HAL Id: halshs-03067554 \\ https://shs.hal.science/halshs-03067554}

Preprint submitted on 15 Dec 2020

HAL is a multi-disciplinary open access archive for the deposit and dissemination of scientific research documents, whether they are published or not. The documents may come from teaching and research institutions in France or abroad, or from public or private research centers.
L'archive ouverte pluridisciplinaire HAL, est destinée au dépôt et à la diffusion de documents scientifiques de niveau recherche, publiés ou non, émanant des établissements d'enseignement et de recherche français ou étrangers, des laboratoires publics ou privés. 
UMR 5824

93. chemin des Mouilles 69130 Ecully - France

Maison de IUniversité, Bâtiment B 10, rue Trefilerie 42023 Saint-Etienne cedex $02 \cdot$ France http://www.gate.cnis.fr gate@gate.cnrs.fr

\title{
A General and Efficient Method for Solving Regime- Switching DSGE Models
}

\author{
Julien Albertini, Stéphane Moyen
}

\begin{abstract}
:
This paper provides a general representation of endogenous and threshold-based regime switching models and develops an efficient numerical solution method. The regime-switching is triggered endogenously when some variables cross threshold conditions that can themselves be regime-dependent. We illustrate our approach using a RBC model with state-dependent government spending policies. It is shown that regime-switching models involve strong non linearities and discontinuities in the dynamics of the model. However, our numerical solution based on simulation and projection methods with regime-dependent policy rules is accurate, and fast enough, to efficiently take into all these challenging aspects. Several alternative specifications to the model and the method are studied.
\end{abstract}

\section{Keywords:}

Regime-switching, RBC model, simulation, accuracy

JEL codes:

E3, J6 


\title{
A General and Efficient Method for Solving Regime-Switching DSGE Models*
}

\author{
Julien Albertini ${ }^{\dagger} \quad$ Stephane Moyen ${ }^{\ddagger}$
}

October 16, 2020

\begin{abstract}
This paper provides a general representation of endogenous and thresholdbased regime switching models and develops an efficient numerical solution method. The regime-switching is triggered endogenously when some variables cross threshold conditions that can themselves be regime-dependent. We illustrate our approach using a RBC model with state-dependent government spending policies. It is shown that regime-switching models involve strong non linearities and discontinuities in the dynamics of the model. However, our numerical solution based on simulation and projection methods with regimedependent policy rules is accurate, and fast enough, to efficiently take into all these challenging aspects. Several alternative specifications to the model and the method are studied.
\end{abstract}

Keywords: Regime-switching, RBC model, simulation, accuracy.

\section{JEL Classification: E3, J6}

\footnotetext{
${ }^{*}$ We are grateful to Klaus Adam, Tom Holden and Lilia Maliar for helpful suggestions. All errors are ours. The paper represents the authors' personal opinions and does not necessarily reflect the views of the Deutsche Bundesbank.

${ }^{\dagger}$ Corresponding author. Email address: Julien.Albertini@univ-lyon2.fr , Univ Lyon, Université Lumière Lyon 2, GATE UMR 5824, F-69130 Ecully, France

${ }^{\ddagger}$ Email address: stephane.moyen@bundesbank.de, Deutsche Bundesbank.
} 


\section{Introduction}

Regime-switching (RS thereafter) models have become increasingly popular in macroeconomic modelling. They encompass models with occasionally binding constraints (financial constraints, default risks, zero lower bound), structural changes, rare disasters, changes in monetary and fiscal policies, etc. The RS is triggered exogenously (Markov process) or endogenously when some variables cross threshold conditions. In the latter case the common practice is to consider that the condition according to which the economy changes regime is the same than the one governing the switch back, implying a symmetry. However, the switching condition may be asymmetric. For instance, a fiscal policy may be triggered when output falls below a threshold value but may be offset, or prolonged, as output reaches a different, higher or lower, threshold. In that case, the RS is asymmetric, and the threshold condition depends on the regime in which the economy stands. The symmetric $\mathrm{RS}$ is, in fact, a special case of the asymmetric RS. The major problem with asymmetric RS is that not only the policy rules are non-differentiable but they are also discontinuous. This feature may strongly distort agents' expectations as well as their decision rules. Solving DSGE models with this asymmetric RS is challenging. Indeed, the switching condition being regime-dependent, the regime becomes a discrete state variable. This paper provides a general representation of endogenous and threshold-based RS (TBRS) models and develops an efficient numerical solution method, that takes into account all the aforementioned non-linearities when computing the expectations and the decision rules.

The expansion of RS models can be explained by their ability to cover a broad variety of issues. Models with occasionally binding constraints like the borrowing constraint or the ZLB constraint in the New Keynesian model are two prominent examples. RS models also belong to models with timevarying parameters. A first branch of this literature uses Markov processes to generate changes in parameters' value like Leeper \& Zha (2003), Sims \& Zha (2006), Chung et al. (2007), Svensson \& Williams (2009), Farmer et al. (2009), Liu et al. (2011), Bianchi (2013). These studies take advantage of the Markovian representation which allows to produce infrequent and long-lived changes in parameters or in the policy rules. The process being exogenous, linear approximation methods can be used to solve and simulate the model.

A second branch of this literature uses endogenous transition probabilities to describe the RS like in Barthélemy \& Marx (2017, 2019), Binning \& Maih (2017), Boneva et al. (2018) and Benigno et al. (2020). The probabilities are state-dependent and time-varying involving that the RS is determined by the variables of the model. It provides an appealing framework to study (but 
not limited to) monetary policy and financial crisis regimes. In addition, perturbation methods can be applied to derive an approximated solution, provided that the model and the transition probabilities are smooth enough ${ }^{1}$. An alternative modelling of endogenous time-varying parameters is proposed by Canova et al. (2020). They consider DSGE models with smoothly evolving parameters according to a differentiable function of the state variables. They show that models with such time-varying parameters improve the fit of the models and overcome important identification issues implied by constant parameters.

The contribution of our paper is threefold. First, we consider thresholdbased RS where the switch occurs exactly when the threshold condition is satisfied without any need for transition probabilities. The RS generates strong non-linear dynamics as it occurs abruptly. This aspect is important because RS affects agents' expectations in a non-smooth manner, thereby affecting the model's outcome. We then further generalize our approach to encompass many other form of RS models featuring smoother transitions. More precisely, we relax the assumption of a clear cutoff by introducing uncertainty in the threshold condition. This allows to deal with uncertainty and credibility issues of economic policies. Also, controlling for the degree and persistence of uncertainty surrounding the threshold condition can provide a flexible way to capture various, more or less smooth, RS dynamics. In a recent paper, Chang et al. (2019) also study TBRS models, aiming at developing an efficient estimation algorithm. They build on the piecewise linear modelling approach of Chang et al. (2017). Closer to us is the important early contribution of Davig et al. (2006). They modeled and studied, using a nonlinear projection solution approach, an endogenous TBRS monetary policy rule, where the latter parameters change as inflation crosses a certain threshold.

As our second major contribution, we extend these approaches by also considering asymmetric, potentially multiple, TBRS. This characteristic may help studying various policy instruments, monetary, fiscal, or macroprudential, that are triggered following a specific set of threshold conditions but are muted according to a different one. Our study is then closely related to $\mathrm{Bi}$ et al. (2013) who consider regime-dependent fiscal consolidation policies. In their model, when the government debt crosses the fiscal limit, fiscal authorities implement a fiscal consolidation. The effective fiscal limit, which governs the RS, is drawn from a distribution. As all endogenous variables are forwards, it turns out that the distribution of fiscal limits can be pre-computed from a Markov Chain Monte Carlo simulation. Consequently, the fiscal limit

\footnotetext{
${ }^{1}$ Barthélemy \& Marx (2017) discuss such conditions.
} 
is time-varying but not regime-dependent which is precisely what we tackle in our study.

Third, we provide an efficient numerical solution for solving this class of DSGE model based on a global approximation ${ }^{2}$.

Solving this class of models is challenging due to the non linearities and the discontinuities in the policy rules. While perturbation methods are fast, they are clearly not appropriate for solving asymetric threshold-based RS models. We then use a projection method that consists in approximating the policy rules by orthogonal polynomials. These methods are known to delivers substantial accuracy in the solution but have often been blamed for their computational costs. We take advantage of the recent improvement from Maliar \& Maliar (2015) to speed up the numerical solution. Their technique called generalized stochastic simulation algorithm (GSSA henceforth) consists in approximating the policy rules on a representative set of points obtained from stochastic simulations. The model is solved only in the area of the state-space visited in equilibrium and it drastically reduces the costly evaluation of the expectation functions thanks to a grid reduction technique ( $\varepsilon$-distinguishable set, or EDS thereafter). We adapt their solution method to RS models. In particular, we consider regime-dependent policy rules which are evaluated on an adaptive grid defined as a collection of regime-clustered points. Each policy rule is evaluated on the area of the state-space that belongs to the corresponding regime. The GSSA produces an accurate and fast solution in all different specifications of the models including kinks, discontinuities, large shocks and multiples regimes.

The paper is organized as follows. We present a general formulation for the RS model and the numerical solution method in Section 2. An application to a RBC model with a regime-dependent government spending policy is then presented in Section 3. Section 4, is devoted to the alternative specifications of the model or of the solution method. Section 5 concludes. We provide a supplementary appendix with an alternative representation of the RS, a detailed description of the algorithms and additional simulations.

\footnotetext{
${ }^{2}$ Methods for solving deterministic models are fast, easy to implement (like for instance with Dynare software package), and are able to manage accurately strong non-linearities. In models that are fairly linear and under the assumption that the shocks are relatively small in magnitude the outcomes from deterministic solution methods is often similar to the ones from stochastic solution methods. In non-linear model however, the deterministic solution may be dramatically different from the stochastic one (see Nakata (2017) for an example with the ZLB in New Keynesian models). Here we are interested in the stochastic method in models characterized by RS which involves important non-linearities.
} 


\section{The RS model}

We consider the standard problem of an agent deciding on the path of a set of control variables, to maximize the discount sum of its future payoffs, subject to a set of constraints. Before deriving the optimization problem we describe the $\mathrm{RS}$ rule.

\subsection{Regime-switching rule}

In the RS model some parameters are regime-dependent and regimes evolve endogenously according to a specific rule. To begin, we denote by $s_{t}$ the regime at time $t$. $s_{t}$ has a finite discrete support $s_{t}=\{1, \ldots, N\}$ where $N$ stands for the number of regimes in the economy. $p$ is the set of parameters characterizing the model. Parameters are regime-dependent. We use the formulation $p\left(s_{t}\right)=p_{1}\left(s_{t}\right), p_{2}\left(s_{t}\right), \ldots, p_{N}\left(s_{t}\right)$ to define the set of parameters when the regime $s_{t}$ applies. The regimes change according to threshold-based rules i.e. when a variable is below or above a threshold. When the number of regimes is higher than two, we consider that the changes in regime are triggered when the endogenous variable lies in a specific interval. The novelty of our approach is that the thresholds are themselves regime-dependent. The thresholds governing the switch from one regime to another may differ depending on the initial regime. Furthermore, there can be several thresholdbased rule, meaning that the changes in the regime are triggered by multiple conditions. For the sake of exposition, we first present the case where the changes in regime only depend on one endogenous variable and later generalize the results to multiple variables.

\subsubsection{One-variable condition}

Denote by $v_{t}$ the variable on which the threshold condition depends. There is no restriction on the variable governing the regime switch. It can be a state, a control, an exogenous variable or a combination of several. We defined by $\underline{v}\left(s_{t}\right)=\left\{\underline{v}_{1}\left(s_{t}\right), \ldots, \underline{v}_{N+1}\left(s_{t}\right)\right\}$ a set of $N+1$ threshold parameters conditional to the initial regime $s_{t}$, and make two assumptions:

(i) The extremas of $\underline{v}$ encompasse all feasible decisions $v_{t}$, that is $v_{t} \in$ ]$\underline{v}_{1}\left(s_{t}\right), \underline{v}_{N+1}\left(s_{t}\right)[, \forall t$

(ii) Conditional on an initial regime $s_{t}$, the thresholds are ordered

$$
\underline{v}_{1}\left(s_{t}\right)<\ldots<\underline{v}_{N+1}\left(s_{t}\right)
$$


Assumption (i) implies that the regime is always defined as long as $v_{t}$ lies within the interval $\left[\underline{v}_{1}(s), \underline{v}_{N+1}(s)\right], \forall s$ or is in the ergodic set. Assumption (ii) simplifies the representation of the law of motion for the regime $s_{t}$. It is worth mentioning that the ordering of the thresholds does not imply any ordering for the value of the parameter $p(1), p(2), \ldots, p(N)$. The law of motion of the regime writes:

$$
s_{t}=\sum_{n=1}^{N} n \times \mathbb{1}_{\left\{v _ { t } \in \left[\underline{v}_{n}\left(s_{t-1}\right), \underline{v}_{n+1}\left(s_{t-1}\right)[\}\right.\right.}
$$

The thresholds are themselves regime-dependent. We label this setup as the asymmetric $R S$, as the cutoff that governs the RS depends on the initial regime. By contrast, the case when the thresholds governing the changes in the regime are independent from the previous regime is labelled as Symmetric $R S$. In the latter, the entry and the exit from the regime are based on the same cutoff. It is worth mentioning that the asymmetric $\mathrm{RS}$ is a general formulation of threshold-based RS (TBRS) as it nests the symmetric case. An important implication is that in the asymmetric $\mathrm{RS} s_{t}$ is a state variable. In the symmetric RS it is not.

We draw the readers' attention on the timing of event. It must be rigorously defined to avoid time inconsistency between variables and regimedependent parameters. For instance, the variable $v_{t}$ which serves to determine today's regime $s_{t}$, can not be calculated if it depends on some parameters $p\left(s_{t}\right)$ that are themselves regime-dependent. In our timing of events the variable $v_{t}$ is determined at the very beginning of the period and its value is not conditional on the regime $s_{t}$ (or on the parameters $p\left(s_{t}\right)$ ). It can however depends on $s_{t-1}$. An alternative could be that the parameters in period $t$ are based on the regime of the previous period.

\subsubsection{Multiple variables condition}

We now consider the case where several endogenous variables govern the RS. We denote $\left\{v_{t}^{1}, \ldots, v_{t}^{L}\right\}$ the set of variables on which a threshold condition applies and $N_{1}, \ldots, N_{L}$ the number of regime for each variable $\ell=1, \ldots, L$. $\underline{v}^{\ell}\left(s_{t}\right)=\left\{\underline{v}_{n \ell}^{\ell}\left(s_{t}\right), \ldots, \underline{v}_{N_{\ell}+1}^{\ell}\left(s_{t}\right)\right\}$ corresponds to the set of ordered threshold parameters that belongs to the variable $v_{t}^{\ell}$, conditional on the initial regime $s_{t-1}$. The law of motion for $s_{t}$ writes:

$$
s_{t}=\sum_{n_{1}=1}^{N_{1}} \ldots \sum_{n_{L}=1}^{N_{L}} \xi\left(n_{1}, \ldots, n_{L} ; N_{1}, \ldots, N_{L}\right) \times\left(\prod_{\ell=1}^{L} \mathbb{1}_{\left\{v_{t}^{\ell} \in\right] \underline{v}_{n_{\ell}}^{\ell}\left(s_{t-1}\right), \underline{v}_{n_{\ell}+1}^{\ell}\left(s_{t-1}\right)[\}}(\text { })\right.
$$


where $\xi$ is the function that sorts the regime number given the elements of the combination and the number of regime per variable $\ell$. Formally it writes:

$$
\xi\left(n_{1}, \ldots, n_{L} ; N_{1}, \ldots, N_{L}\right)=n_{L}+\sum_{i=1}^{L-1}\left[\left(n_{L-i}-1\right) \times \prod_{\ell=L-i+1}^{L}\left(N_{\ell}\right)\right]
$$

For instance, if there are two regimes based on a threshold condition on variable 1 and three regimes based on a threshold condition on variable 2 , the total number of regime is 6 . $\xi$ gives the regime number associated to the element $n_{1}$ and $n_{2}$.

\subsection{TBRS-DSGE models}

The problem of an agent is to choose a sequence of controls $\left\{y_{t}\right\}_{t=0}^{\infty}$ maximizing the expected discount sum of instantaneous payoffs subject to a set of constraints. In a stochastic environment, decisions are taken given the evolution of aggregate shocks. We denote by $z_{t}$ a vector of stationary and exogenous shocks. It satisfies the following representation:

$$
z_{t}=\Phi\left(z_{t-1}, \varepsilon_{t}\right) \quad \text { with } z_{0} \text { given and } \varepsilon_{t} \sim \mathcal{N}\left(\mu, \sum\right)
$$

where $\varepsilon_{t}$ is a vector of i.i.d. structural disturbances with mean vector $\mu$ and covariance matrix $\sum$. Let $x_{t}$ be a vector of endogenous state variables (including the regimes) evolving as follows:

$$
x_{t}=h\left(x_{t-1}, z_{t-1}, y_{t}\right) \text { with } x_{0} \text { given }
$$

The agent's problem takes the following recursive form:

$$
\begin{aligned}
V\left(x_{t-1}, z_{t-1}\right)= & \max _{y_{t} \in \mathcal{D}\left(x_{t-1}, z_{t-1}\right)}\left\{u\left(x_{t-1}, z_{t-1}, y_{t}\right)+\beta E_{t} V\left(x_{t}, z_{t}\right)\right\} \\
& \text { s.t. } 0=\left(x_{t}-h\left(x_{t-1}, z_{t-1}, y_{t}\right)\right)\left(\lambda_{t}\right)
\end{aligned}
$$

where $V($.$) is the optimal value the agent can derive from the maximization$ problem and $u($.$) is the instantaneous payoff function. \mathcal{D}$ corresponds to the set of feasible decisions. $\lambda_{t}$ are the Lagrange multipliers associated to the constraints and $\beta$ is the discount factor. The agent chooses a set of processes $\left\{y_{t}\right\}_{t=0}^{\infty}$ maximizing the value function (6) subject to the law of motion of $x_{t}$ and taking as given the set of processes $\left\{z_{t}\right\}_{t=0}^{\infty}$. The first-order conditions of this problem write:

$$
\begin{aligned}
& u_{y_{t}}^{\prime}\left(x_{t-1}, z_{t-1}, y_{t}\right)=\lambda_{t} h_{y_{t}}^{\prime}\left(x_{t-1}, z_{t-1}, y_{t}\right) \\
& \lambda_{t}=\beta E_{t}\left[\lambda_{t+1} h_{x_{t}}^{\prime}\left(x_{t}, z_{t}, y_{t+1}\right)-u_{x_{t}}^{\prime}\left(x_{t}, z_{t}, y_{t+1}\right)\right]
\end{aligned}
$$




\section{$2.3 \quad$ Numerical method}

\subsubsection{General formulation of the solution}

The general solution of this family of discrete-time rational expectations models with RS writes:

$$
E_{t}\left(R\left(\boldsymbol{x}_{t-1}, y_{t}, \boldsymbol{x}_{t}, y_{t+1}\right)\right)=0
$$

where $\boldsymbol{x}_{t}=\left\{x_{t}, z_{t}\right\}$ is the vector of state variables. $R($.$) is the residual$ function defined by the FOC (8) and the evolution of the state variables. It is defined as follow:

$$
R(.)=\left[\begin{array}{l}
u_{y_{t}}^{\prime}\left(\boldsymbol{x}_{t-1}, y_{t}\right)=\lambda_{t} h_{y_{t}}^{\prime}\left(\boldsymbol{x}_{t-1}, y_{t}\right) \\
\lambda_{t}-\beta \mathcal{E}_{t+1} \\
\mathcal{E}_{t}-\left[\lambda_{t} h_{x_{t-1}}^{\prime}\left(\boldsymbol{x}_{t-1}, y_{t}\right)-u_{x_{t-1}}^{\prime}\left(\boldsymbol{x}_{t-1}, y_{t}\right)\right] \\
x_{t}-h\left(\boldsymbol{x}_{t-1}, y_{t}\right) \\
z_{t}-\Phi\left(z_{t-1}, \varepsilon_{t}\right)
\end{array}\right]
$$

The solution of the above problem consists in expressing the set of control variables $y_{t}$ as a function of the state variables $\boldsymbol{x}_{t}$ and $s_{t}$.

$$
y_{t} \equiv g\left(\boldsymbol{x}_{t-1} ; p\left(s_{t}\right)\right)
$$

In most DSGE models, the function $g($.$) can not be computed analytically,$ so does the residual function $R($.$) . The projection method consists in us-$ ing Chebyshev polynomials as approximation functions. However, (1) has a discrete support $\{1,2, \ldots, N\}$ which involves non-differentiable policy rules. Spectral methods based on smooth approximation functions are likely to generate inaccuracy of the approximation at the kinks and when the regime switches. In order to circumvent this difficulty, we consider regime-dependent decision rules. We denote by $\Theta(s)=\left\{\theta_{1}(s), \ldots, \theta_{m}(s)\right\}$ the set of coefficients of the approximation function in regime $s$. The approximation function for (11) is:

$$
y_{t}=P\left(\boldsymbol{x}_{t} ; \Theta\left(s_{t}\right)\right)
$$

The general idea of the projection method is to find the set of coefficients $\Theta($.$) minimizing (10). We make this step through OLS regression. Formally$ it writes:

$$
\hat{\Theta}\left(s_{t}\right)=\arg \min _{\Theta\left(s_{t}\right)} \int_{\boldsymbol{x}_{t}} R\left(\boldsymbol{x}_{t} ; \Theta\left(s_{t}\right)\right)^{2} d \boldsymbol{x}_{t}
$$


where $R\left(\boldsymbol{x}_{t} ; \Theta\left(s_{t}\right)\right)$ is the residual equation (10) given that $y_{t}$ is approximated by a polynomial function of the state variables.

In our numerical approach, there are as many approximation functions as we have regimes in the economy. In the spirit of Christiano \& Fisher (2000), the global solution is a piecewise interpolation of approximation functions. The connections between approximation functions are endogenously determined by the RS conditions.

\subsubsection{Algorithm}

Following Maliar \& Maliar (2015), we extend the generalized stochastic simulation algorithm (GSSA) which combines deterministic integration and stochastic simulations. The general idea consists in simulating the model and projecting the polynomials on a set of representative simulated points. Integrals are evaluated using Gauss Hermite quadratures only on the representative points to reduce the computational burden.

As mentioned by the authors, this hybrid method is fast and accurate for two reasons. First, thanks to the simulation techniques, the solution is only evaluated on a set of points that are visited in equilibrium. It contrasts with standard projection methods in which the polynomial is projected on a multidimensional fixed grid of points which resembles a hypercube (or hyperrectangle). In most DSGE models with normally distributed shocks the ergodic set resembles a hyper-sphere or a hyper-ellipsoid. The fixed grid then cover a larger fraction of the ergodic set. Consequently, many grid points are never visited in equilibrium. Judd et al. (2011) show in a two dimensional case that the ergodic set is at least $21 \%$ smaller than the square. This "useless" area is all the more larger than the dimension of the state space increases. Contrary to standard fixed grid method ${ }^{3}$, simulation techniques belongs to endogenous and adaptive grid methods. The major advantage is that they precisely focus on the relevant area of the state-space, thereby increasing the accuracy.

The second reason that motivate the use of this method is that it is relatively fast. Indeed, we borrow from Maliar \& Maliar (2015) the stateof-the-art techniques of representative points using the $\varepsilon$ - distinguishable set (EDS) method. Their strategy consists in eliminating points that are redundant i.e. simulated points that are not useful for evaluating the policy rule as they are in the neighbor of other points. One important advantage of the representative points technique is that the costly evaluation of integrals

\footnotetext{
${ }^{3}$ With the exception of Judd et al. (2014) where a Smolyak hypercube is adapted to a solution domain of a given economic model and Brumm \& Scheidegger (2017) who use adaptive sparse grid methods.
} 
is alleviated. The computation of expectations using quadratures techniques is only made on the EDS and not on the whole simulated points. Since a very small number of representative points is needed to obtain a decent accuracy, the algorithm is thus relatively fast.

The novelty of our approach is that we consider piecewise interpolated polynomials projected on regime-clustered points obtained by stochastic simulations. More precisely, we consider one policy rule per regime. The statespace on which the policy rules are projected is simulated and partitioned according to the regime. The policy rules are interpolated when calculating expectation functions. This strategy saves computational times and greatly improves accuracy because we evaluate the polynomial coefficients only in the state-space that is visited in simulations and that belongs to the regime. Consequently, a collection of smooth approximation function can handle the extreme non linearities imposed by the RS specification. We describe below the steps of the general algorithm and advise the reader to refer to the supplementary appendix for a detailed version applied to the RBC model.

\section{Algorithm}

Step 1 - Initialization. Set $i=0$ the i-th iteration. Choose $d$, the Chebyshev polynomial order and the simulation length $T$. Initialize the coefficients $\Theta(n)^{i}, n=1, \ldots N$ using OLS regression on a stochastic simulation of $T$ periods computed from the solution of a perturbation method ${ }^{4}$.

Step 2 - Stochastic simulations. Draw $\left\{\varepsilon_{t}\right\}_{t=0}^{T}$ and compute a stochastic simulation to get $\left\{\boldsymbol{x}_{t}\right\}_{t=0}^{T}$ using Equation (4) and (5) and to get $\left\{y_{t}\right\}_{t=0}^{T}$ using Equation (12).

Step 3 - Representative points. [Maliar \& Maliar (2015), section 2.2.2]. Set the parameter $\varepsilon_{n}, n=1, \ldots, N$. Define $X_{n}=\left\{\boldsymbol{x}_{t} \mid s_{t}=n\right\}, n=1, \ldots, N$ a partition of the simulated series for which the regime is equal to $n$. Denote by $\mathcal{P}_{n}$ an empty set such that $\mathcal{P}_{n}=\{\varnothing\}$. Compute the Euclidian distance between the principal component of $X_{n}$. Eliminate from $X_{n}$ all points for which the distance is $<\epsilon_{E D S}$ (the maximal distance between points) and add them to $\mathcal{P}_{n}$.

Step 4 - Expectation. Set Gauss-Hermite quadratures nodes $\boldsymbol{e}$ and weights $\omega$. For each representative points of $\mathcal{P}_{n}$, compute the integral $\beta E_{t} \mathcal{E}_{t+1}$ of the Euler equation. Given the evaluation of the expectation functions,

\footnotetext{
${ }^{4}$ As the perturbation method can not handle the RS, we simply assume that $s_{t}$ is constant and equal to one over the whole simulation.
} 
pin down the estimated value of the control variables $\left(\hat{\lambda}_{t}, \hat{y}_{t}\right)$ at each representative points from Equation (8) using a root finding procedure.

Step 5 - Estimate coefficients. Construct the residual function $R($.$) and$ find the coefficient that minimize this function, in line with objective from Equation (13). Iterate on Step 2 to 5 until achieving the target minimum of the residual function.

\section{Application to a RBC model}

We present the canonical RBC model and introduce non-separable government spending. The value of the government spending switches between two regimes according to a threshold-based rule defined by the level of output.

\subsection{The model}

The representative household chooses a consumption path to maximize its intertemporal utility:

$$
\begin{aligned}
\max _{\left\{c_{t}, k_{t+1}\right\}_{t=0}^{\infty}} & E_{t} \sum_{t=1}^{\infty} \frac{\left(c_{t}+\rho g_{t}\left(s_{t}\right)\right)^{1-\sigma}-1}{1-\sigma} \\
\text { s.t. } & c_{t}+k_{t}=(1-\delta) k_{t-1}-g_{t}\left(s_{t}\right) \\
& y_{t}=\exp \left(z_{t}\right) k_{t-1}^{\alpha} \\
& k_{0} \quad \text { is given }
\end{aligned}
$$

where $\delta$ is the depreciation rate of capital, $y_{t}$ is output and $g_{t}$ is the regimedependent government spending level ${ }^{5}$. Furthermore, $z_{t}$ is a stochastic process:

$$
z_{t}=\rho_{z} z_{t-1}+\varepsilon_{t} \quad \text { with } \varepsilon_{t} \sim \mathcal{N}\left(0, \sigma_{z}^{2}\right)
$$

\footnotetext{
${ }^{5}$ In this model agents take as given the law of motion of $s_{t}$ and the underlying parameter variations. One could assume that agents internalize the effects their decisions on the regime $s_{t}$ by considering explicitly Equation (1) as a constraint in the program. This internalization effects is studied in Canova et al. (2020) where some parameters of their model are state-dependent. They use smooth polynomial functions to express the parameters as a function of the state variables. They show that there is a second (endogenous) effect that results from the derivative of the parameters with respect to the state variables. However, in a RS setup, the law of motion for $s_{t}$ is non differentiable and discontinuous, making such derivations difficult. While those aspects may be of a great importance we leave them open for future research and restrain our analysis to tractable problems that takes the form of (14).
} 
The first-order conditions of the above program are:

$$
\begin{aligned}
& \lambda_{t}=\left(c_{t}+\rho g_{t}\left(s_{t}\right)\right)^{-\sigma} \\
& \lambda_{t}=\beta E_{t} \lambda_{t+1}\left(\alpha \exp \left(z_{t+1}\right) k_{t}^{\alpha-1}+1-\delta\right)
\end{aligned}
$$

$\lambda_{t}$ is the Lagrange multiplier associated to the budget constraint. In our setup the regime evolves according the threshold-based rule on output. Let $\underline{y}\left(s_{t-1}\right)$ be the output threshold governing the regime switching which can be asymmetric. The set of ordered threshold for output is:

$$
\underline{y}\left(s_{t-1}\right)=\left\{\underline{y}_{1}\left(s_{t-1}\right), \underline{y}_{2}\left(s_{t-1}\right), \underline{y}_{3}\left(s_{t-1}\right)\right\}
$$

Denote by $N_{y}=2$ the number of regimes from the condition on output, the law of motion for the regime is:

$$
s_{t}=\sum_{n_{1}=1}^{N_{y}} n_{1} \times \mathbb{1}_{\left\{y_{t} \in\right] \underline{y}_{n_{1}}\left(s_{t-1}\right), \underline{y}_{n_{1}+1}\left(s_{t-1}\right)[\}}
$$

For the sake of simplicity we assume that $\underline{y}_{1}()=.-\infty, \underline{y}_{3}()=.+\infty$. The law of motion of the regime can be simplified to:

$$
s_{t}=\left\{\begin{array}{lll}
1 & \text { if } & y_{t}>\underline{y}\left(s_{t-1}\right) \\
2 & \text { if } & y_{t} \leq \underline{y}\left(s_{t-1}\right)
\end{array}\right.
$$

In the RS environment $g_{t}$ is linked to the regime $s_{t}$ and there exist two regimes:

$$
g_{t}\left(s_{t}\right)=\left\{\begin{array}{lll}
g_{1} & \text { if } & s_{t}=1 \\
g_{2} & \text { if } & s_{t}=2
\end{array}\right.
$$

The equilibrium is a 6 -tuple $\left\{k_{t}, z_{t}, s_{t}, c_{t}, y_{t}, g_{t}\right\}$ given by the system of six equations (15)-(23).

\subsection{Quantitative evaluation}

\subsubsection{Calibration}

The model is calibrated to quarterly frequencies. From the RBC literature we consider fairly standard value for the parameters (Table 1 ). We make a very stylized approximation to parameterize the change in government spending. We aim at reproducing the increase in government spending similar to the American Reinvestment and Recovery Act. Recall that this is more an illustrative example than a precise representation of how the fiscal regimes 
are actually implemented. We target the ratio of government spending on output at $20 \%$ in regime 1 . We consider that in regime $2 g(2) / y=22 \%$. The fiscal expansion program has been triggered when output felt by $4 \%$ and stopped when output was around $2 \%$ point higher than its potential level. We thus consider $y(1)=0.96 y$ and $y(2)=1.02 y$. From Fève et al. (2013), the parameter $\rho$ that controls for the substitutability/complementarity between private consumption and public spending is estimated to be around -0.6 on average across the different specification considered. The size of the technological shock is set to 0.025 , a value that is higher than conventional $\operatorname{AR}(1)$ estimates but that allows for sizeable fluctuations of output and persistent regime-switching.

Table 1: Calibration

\begin{tabular}{lcc}
\hline Variables & Symbol & Value \\
\hline Discount factor & $\beta$ & 0.99 \\
Capital share & $\alpha$ & 0.33 \\
Depreciation rate & $\delta$ & 0.025 \\
Risk aversion & $\sigma$ & 2 \\
Persistence shock & $\rho_{z}$ & 0.90 \\
Substitutability of private & $\rho$ & -0.60 \\
consumption for spending & & $0.20 y$ \\
Government spending R1 & $g(1)$ & $0.22 y$ \\
Government spending R2 & $g(2)$ & $0.96 y$ \\
Threshold R1 $\rightarrow$ R2 & $\underline{y}(1)$ & $1.02 y$ \\
Threshold R2 $\rightarrow$ R1 & $\underline{y}(2)$ & \\
\hline
\end{tabular}

R1: Regime 1, R2: Regime 2. y is the deterministic steady state of output

\subsubsection{Solution implementation}

In this section, we show how the solution method is implemented and how accurate is the algorithm.

Simulated grid Figure 1 shows the implementation of the GSSA with regime-clustered points. Panel (a) displays all points from the stochastic simulations. Panel (b) shows which points are kept when the simulated state variables are passed through the EDS procedure. The EDS technique consists in selecting only representative points by calculating the distance between the points using principal components. The criterion $\left(\epsilon_{E D S}=0.4\right)$ used for eliminating points that are to close to each other is chosen to get a 
minimal number of representative points. The points displayed in Panel (c) correspond to those used to compute the expectation functions. The number of points in each regime is nearly identical, around 200 grid points in each regime. As expected we observe an overlap between the two regimes. Due to the asymmetries in the regime-switch, this area of the state space can belongs to regime 1 or regime 2 .

\section{Figure 1: State-space}

(a) All points

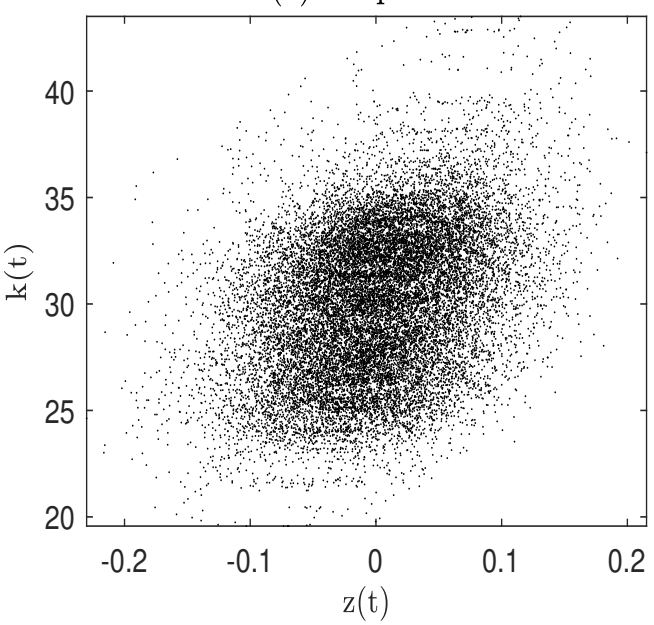

(c) EDS points

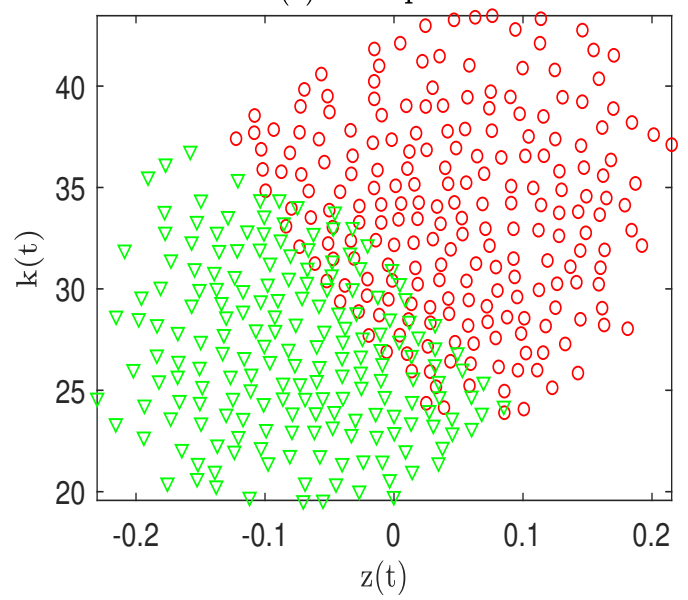

(b) All points vs EDS points

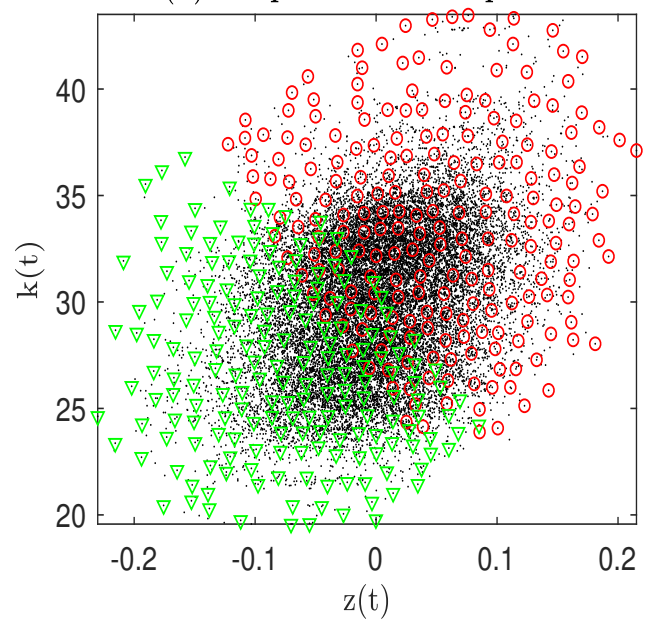

Grid points are computed on a stochastic simulation with 20000 draws from $\varepsilon_{t}^{z}$.

Accuracy and speed When it comes to solution methods, accuracy and speed are two critical issues. For smooth and linear models, linearization and perturbation methods are often preferred since they are extremely fast and 
sufficiently accurate. For nonlinear models, these methods may suffer from spurious approximation. Global approximation methods like projections, $\mathrm{Pa}$ rameterized expectation algorithm, finite elements and value function iteration can handle non-linear models accurately. However, despite considerable improvement in computer chips, these methods are still time-consuming in models characterized by several state variables.

In this section we investigate how accurate and fast is our projection method. We borrow from Judd $(1992,1998)$ the accuracy test that consists in the evaluation of the residuals of the Euler equations calculated on a stochastic simulation. It examines if the policy functions are accurately approximated over two consecutive periods. The idea is to compute the oneperiod optimization error in relation to current consumption. We define the two sides of the Euler equation as:

$$
\begin{aligned}
c_{t} & =P\left(k_{t-1}, z_{t-1} ; \Theta_{c}\right) \\
\hat{c}_{t} & =\left[\beta E_{t} c_{t+1}^{-\sigma}\left(\exp \left(z_{t+1}\right) \alpha k_{t}^{\alpha-1}+1-\delta\right)\right]^{-\frac{1}{\sigma}}
\end{aligned}
$$

Formally, the Euler Equation Errors (EEE thereafter) writes:

$$
E E E=\log _{10}\left(\frac{1}{T} \sum_{t=2}^{T}\left|1-\frac{c_{t}}{\hat{c}_{t}}\right|\right)
$$

The EEE is normalized by the level of consumption in such a way that it furnishes the loss in terms of consumption agents would suffer from using the approximate solution rather than the true solution. As mentioned by Judd (1992), an EEE (in $\log _{10}$ unit) of -3 involves that a person with $\$ 1,000$ of consumption makes at most a one dollar error in current consumption each period. With a value of -4 the error is one dollar per $\$ 10,000$ of consumption. The value -3 is often considered as small and acceptable for a solution method to be accurate. The expectation term is evaluated using Monte Carlo simulations with 10000 draws on each simulated points (not just on EDS points).

Benchmark specification We consider fourth order Chebyshev Polynomials to approximate the consumption function. With two state variables $\left(z_{t}\right.$ and $k_{t-1}$ ) we then have 15 basis functions for each of the two regimes. In order to evaluate the expectation function we use Gauss-Hermite quadratures with 10 nodes. 
Figure 14 shows how EEE are distributed over the visited state-space obtained by simulations. It is shown that the maximum EEE obtained is -3.22 and the average EEE is -4.43 . These values indicate that the consumption error is bellow one dollar per $\$ 1000$ dollar of consumption. The overall accuracy level of our approximation method is then fairly good given the highly non-linear nature of the model. Figure 14 makes it clear that the highest EEE are located at the boarder of the RS cutoff in each regime. When the value of output is close to the threshold governing the RS, the calculation of the expectation functions takes into account this potential switch. This aspect breaks the smoothness of the expectation functions

\section{Figure 2: Euler Equation Errors}

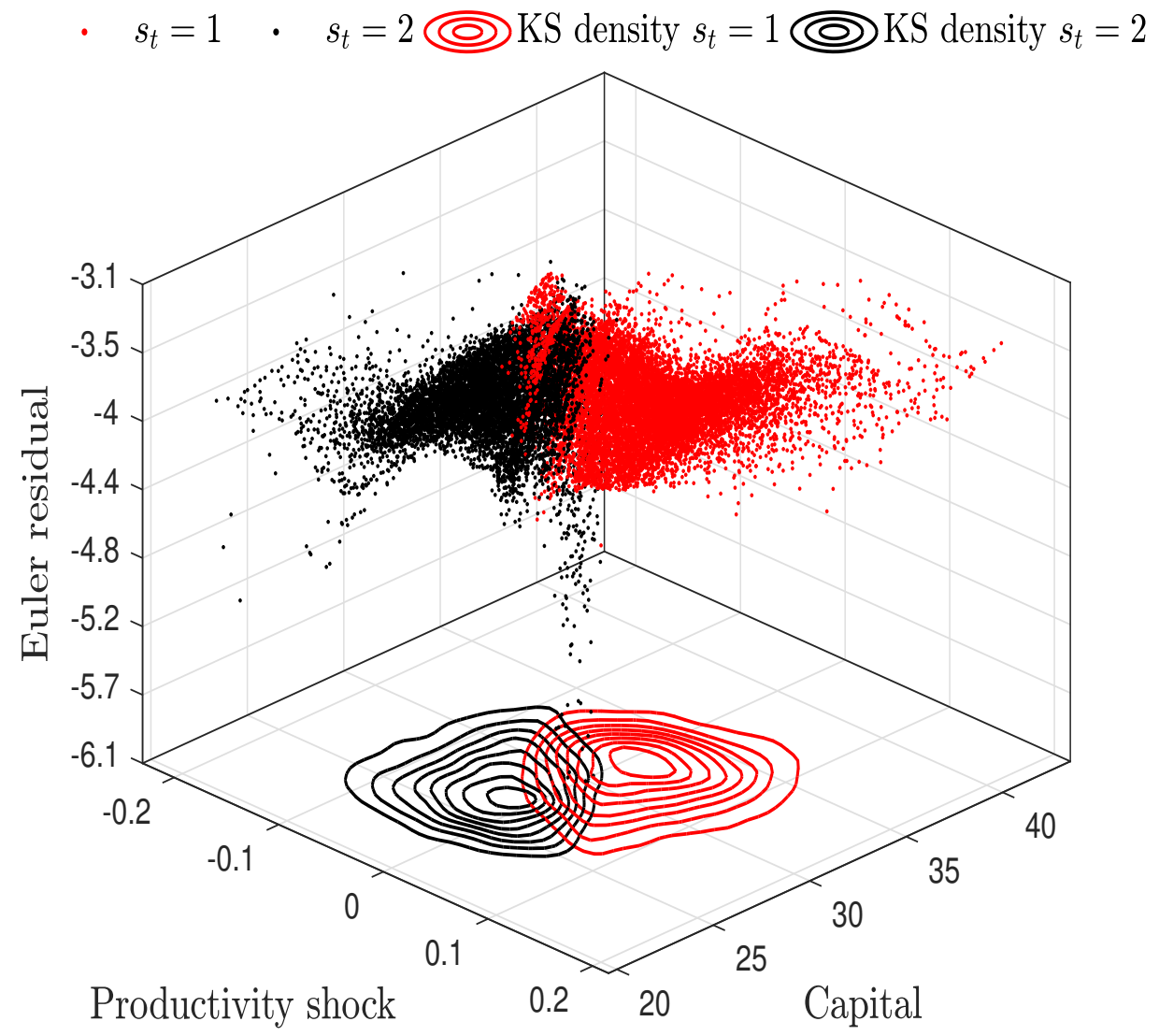

Euler equation errors (in log10 units) are computed on a stochastic simulation with 20000 draws from $\varepsilon_{t}^{z}$. Bivariate smooth Kernel density estimate are displayed for each regime. 
Alternative integration techniques So far we apply the Gauss Hermite quadrature to evaluate the integrals. As they usually apply to smooth function, one may wonder whether this technique is relevant for computing non-smooth function. To do so, we use the Monte Carlo method and the quasi Monte Carlo method when computing the integrals in Step 4 of the algorithm. Let $\Xi\left(\varepsilon_{t+1}^{z}\right)$ be the expectation function. The numerical integration then writes:

$$
\int_{-\infty}^{\infty} \Xi\left(\varepsilon_{t+1}^{z}\right) f\left(\varepsilon_{t+1}^{z}\right) d \varepsilon_{t+1}^{z} \approx \frac{1}{H} \sum_{h=1}^{H} \Xi\left(\tilde{e}_{h}\right)
$$

where $f($.$) is a density function of a Normal distribution. In the Monte$ Carlo algorithm we simply draw each $\tilde{e}_{h}$ from a Normal distribution $\mathcal{N}\left(0, \sigma_{z}^{2}\right)$. For the quasi Monte Carlo integration, we use low-discrepancy sequences to generate points in space in a highly uniform manner. In particular, we use Halton sequences (Halton, 1964) to generate quasi-random point set object.

Table 2 shows that Gauss Hermite quadratures may perform as well as the two types of Monte Carlo method. However, while few nodes are necessary in linear models (Maliar \& Maliar, 2015), our model requires a minimum of 10 nodes to be efficient. The computational time for solving the model with 10 nodes remains largely acceptable as compared to Monte Carlo methods. From a computational time/accuracy ratio the quadratures seem a better option. 
Table 2: Euler Residuals

\begin{tabular}{cccc}
\hline \multirow{2}{*}{ Method } & \multicolumn{2}{c}{ Euler residuals } & Time \\
\cline { 2 - 3 } & $L_{1}$ & $L_{\text {inf }}$ & (in sec) \\
\hline GH quad. & & & \\
3 nodes & -4.17 & -2.66 & 8.5 \\
5 nodes & -4.19 & -2.96 & 8.1 \\
10 nodes & -4.43 & -3.22 & 9.6 \\
\hline MC & & & \\
$10^{2}$ draws & -3.51 & -3.00 & 13.7 \\
$10^{3}$ draws & -4.14 & -3.43 & 19.6 \\
$10^{4}$ draws & -4.21 & -3.50 & 105.2 \\
\hline Quasi MC & & & \\
$10^{2}$ draws & -3.69 & -3.04 & 11.4 \\
$10^{3}$ draws & -3.68 & -3.15 & 24.1 \\
$10^{4}$ draws & -3.69 & -3.16 & 140.4 \\
\hline
\end{tabular}

GH quad. stands for Gauss-Hermite quadratures. MC stands for Monte Carlo methods. In the Quasi $M C$, we use Halton sequences to generate quasi-random point set object and apply inverse transform sampling to get normally distributed shocks. $L_{1}$ : mean Euler residuals. $L_{\mathrm{inf}}$ : maximum Euler residuals. Residuals are computed on each of the 20000 observations (No EDS) from a stochastic simulation with draw on $\varepsilon_{t}^{z}$.

EDS points How many EDS points are necessary to compute the solution accurately? What is the computational time gain? Figure 3 shows how the maximum EEE decreases as the number of EDS grid points increases. The results do not involve a relation between average EEE and the number of EDS points. All in all, it is shown that even with few points the accuracy remains fairly good. EEE are lower than -3 with only 276 points. Not surprisingly, the computational time increases exponentially with the number of EDS points. 


\section{Figure 3: EDS grid point}

(a) Euler residuals

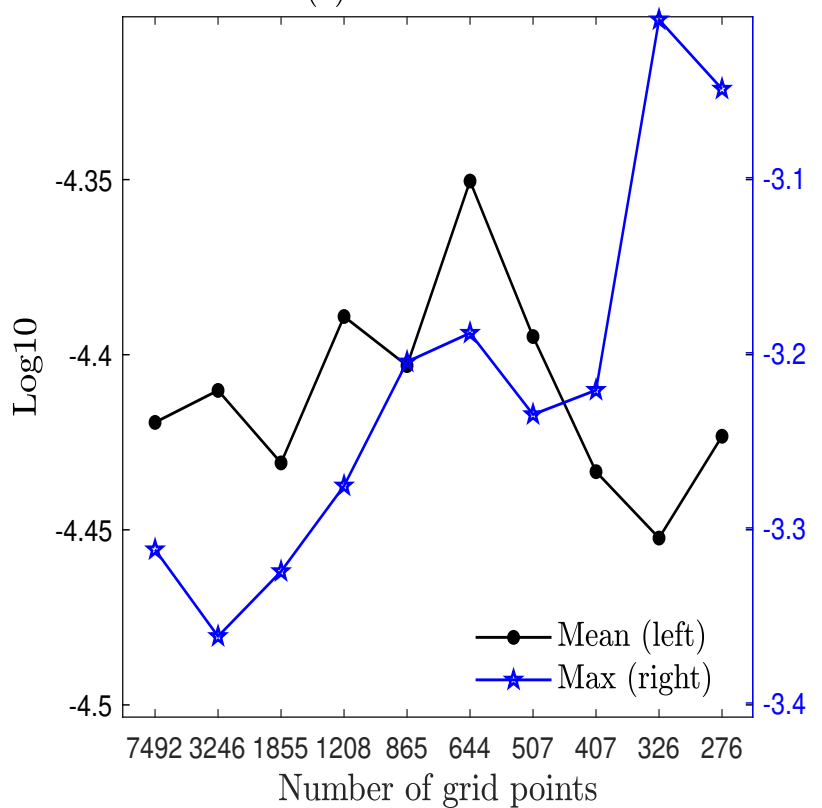

(b) Computational time (in seconds)

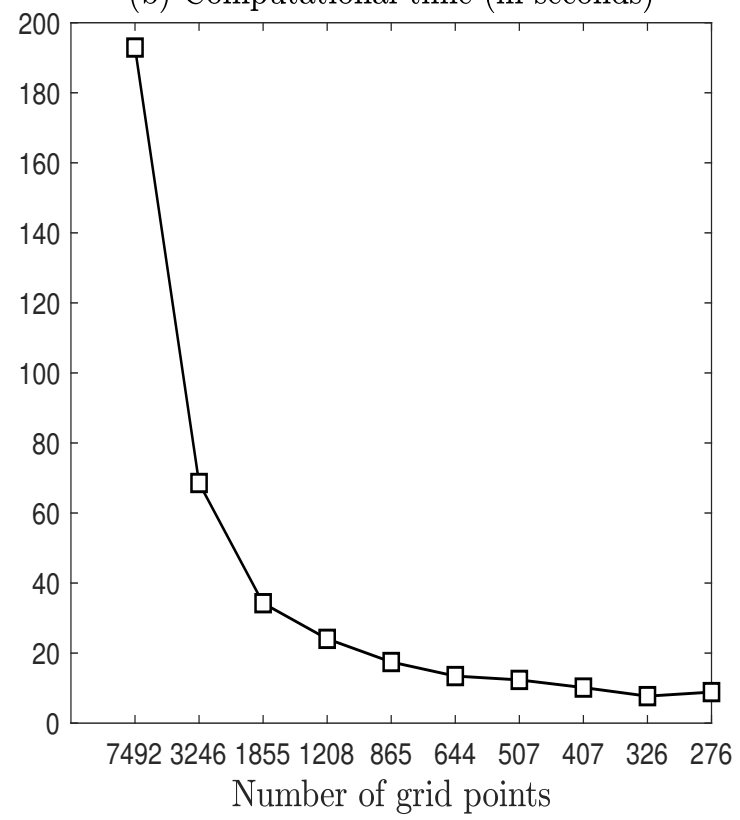

$L_{1}$ mean Euler residuals. $L_{\mathrm{inf}}$ maximum Euler residuals. Residuals are computed on each of the 20000 observations (No EDS) from a stochastic simulation with draw on $\varepsilon_{t}^{z}$ and using Monte Carlo integration with 10000 draw at each time t. The abscise scale is not linear.

Polynomial order The regime-clustered GSSA method splits the policy rule into two regimes. Are the policy rules in each regime non-linear? In other words, do we need high order polynomial? In the benchmark specification, the order of the Chebyshev polynomial is 4, achieving the highest accuracy. Table 3 displays the EEE and the computational time for different order of the polynomial. A polynomial of order two is actually sufficient to achieve a maximum EEE lower than -3. On the contrary a piecewise linear approximation is not accurate enough. In general, low polynomial order are generally faster to compute because there are few basis functions to evaluate which reduces the size of matrices that are inverted in the OLS step to estimate Chebyshev coefficients. In our model, each pieces are not very non-linear but the combination of the two gives rise to a very non-linear model.

If the policy rule in each regime is non-linear one should consider higherorder Chebyshev polynomials. Another possibility from the spectral element 
method (Patera (1984)) is to consider refinement of either, the order of the polynomials, or the number of regime (or elements). The former, known as the $p$-refinement, consists in increasing the order of the approximation. There is no need to choose an order similar for each element. The idea is to consider different polynomial order for each element, depending on the accuracy. The latter method, know as the h-refinement, consists in increasing the number of elements. The idea is to divide the regimes into sub-regime even those regimes are not linked to a specific rule like the one we have on government spending. By doing so, we end up with more policy rules to evaluate but with lower order of the approximation. Lastly, the $h p$-refinement method (Demkowicz et al., 2002; Demkowicz, 2006) combine both and search for the optimal approximation order and the number of elements.

Table 3: Euler Residuals

\begin{tabular}{cccc}
\hline \multirow{2}{*}{ Method } & \multicolumn{2}{c}{ Euler residuals } & Time \\
\cline { 2 - 3 } Order 1 & -3.47 & $L_{\text {inf }}$ & (in sec) \\
Orden & -2.48 & 6.1 \\
Order 2 & -3.87 & -3.08 & 7.8 \\
Order 3 & -4.26 & -3.29 & 6.7 \\
Order 4 & -4.43 & -3.22 & 10.0 \\
Order 5 & -4.27 & -3.14 & 13.2 \\
\hline
\end{tabular}

$L_{1}$ : mean Euler residuals. $L_{\mathrm{inf}}:$ maximum Euler residuals. Residuals are computed on each of the 20000 observations (No EDS) from a stochastic simulation with draw on $\varepsilon_{t}^{z}$.

\subsubsection{Simulations of the model}

Policy rule We now investigate the model's properties. Figure 4 displays the policy rule for consumption as a function of capital (Panel a) and the productivity shock (Panel b). There are two policy rules, one for each regime. As mentioned previously, the policy rules are not extremely non linear in each regime as it is the case for the standard RBC model without RS. The asymmetric aspect of the RS model involves an overlap of the policy rule in some area fo the state-space. For a given value of capital $k_{t-1}$ and the productivity shock $z_{t}$ it is possible to have different level of consumption, depending on the initial regime. This creates discontinuities in the policy rules. 
Figure 4: Policy rules

(a) Consumption

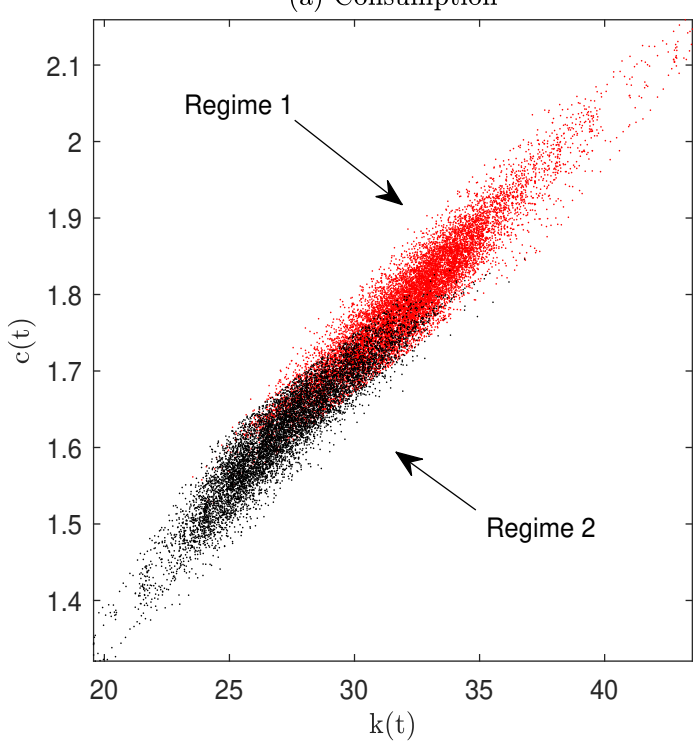

(b) Consumption

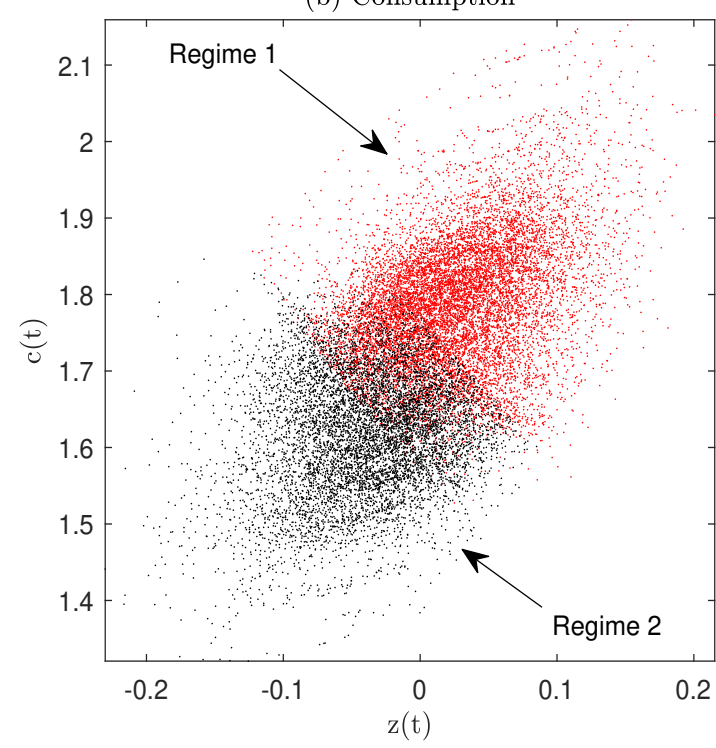

Policy rule $c\left(k_{t-1}, z_{t}\right)$.

Figure 5 shows the simulated path of output and the regime. The economy moves from regime one to regime 2 when output crosses the threshold $\underline{z}(1)$. It switches back only when output increases above $\underline{z}(2)$. This aspect also involves that there exist two stochastic steady states and the economy can move from one another depending on the size of the shock. We further investigate this aspect through impulse response functions. 
Figure 5: Simulated path

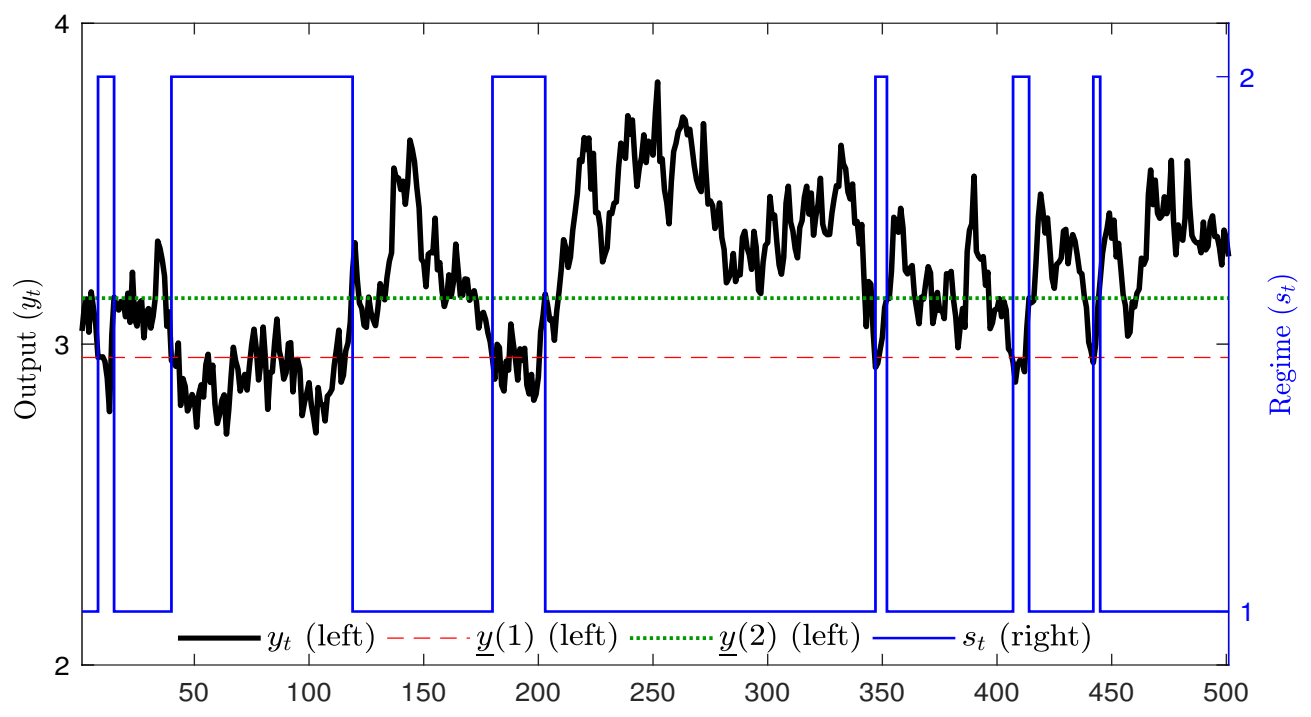

Impulse response functions To investigate the RS along the business cycle, we consider various negative productivity shocks, from $-1 \%$ to $-10 \%$. The initial regime is $s_{0}=1$. The economy remains in regime one when the shock is not large enough to drive output below $4 \%$ of its initial value. In that case the economy come back to its long-run value after the shock. The regime changes from 1 to 2 when the shock is larger. In that case, consumption declines further and so does capital and output. As output does not overcome the threshold that sends the economy back to regime 1 , the temporary shock has permanent effects. As long as no new shock occurs or if a shock occurs with small amplitude, the economy remains in regime 2 where consumption, capital and output are all lower. Our model illustrates the possibility of two different, stable, stochastic steady states around which the economy fluctuates. 
Figure 6: Impulse response functions

(a) Productivity shock $z_{t}$

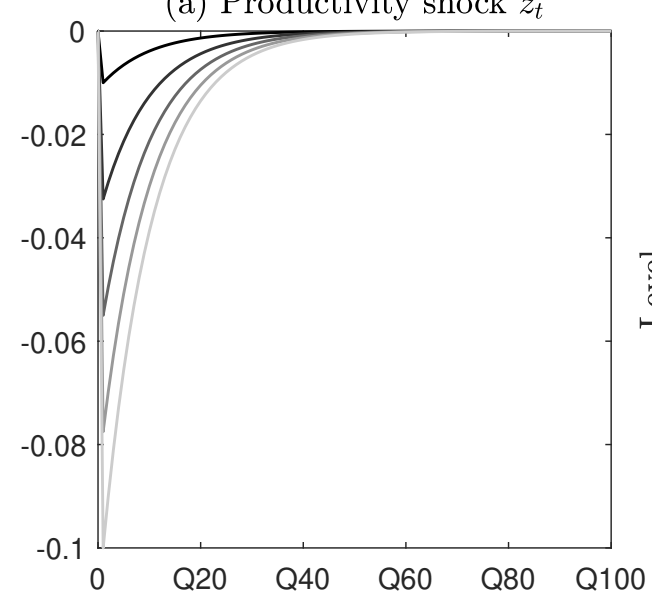

(c) Capital $k_{t}$

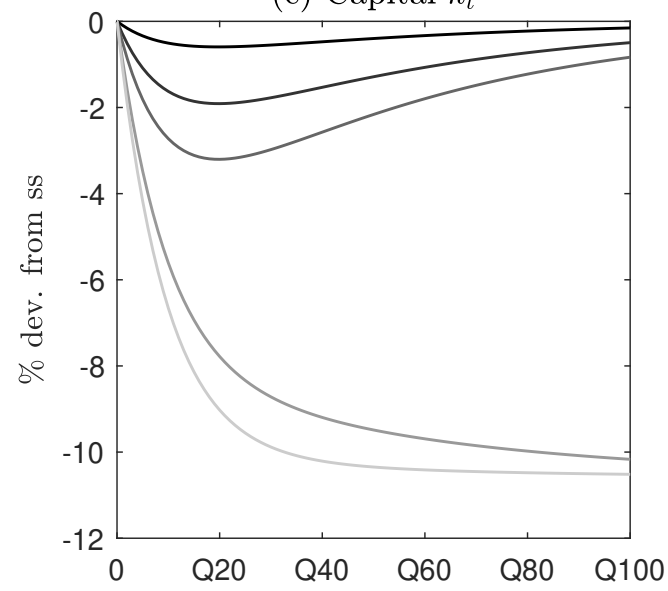

(b) Output $y_{t}$

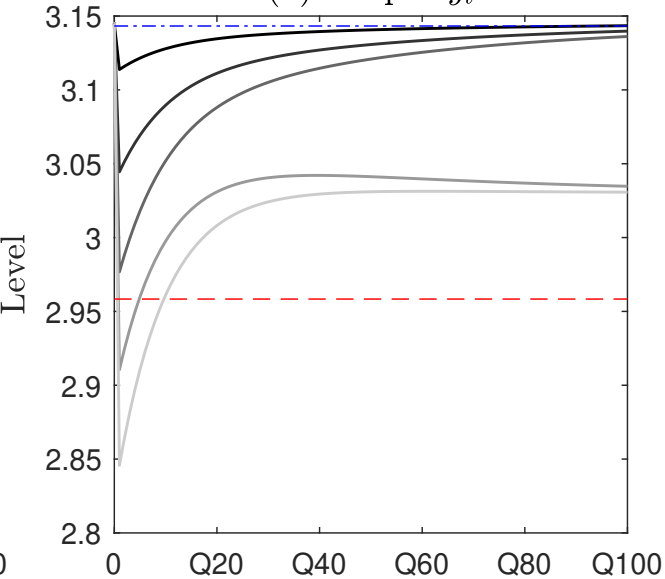

(d) Consumption $c_{t}$

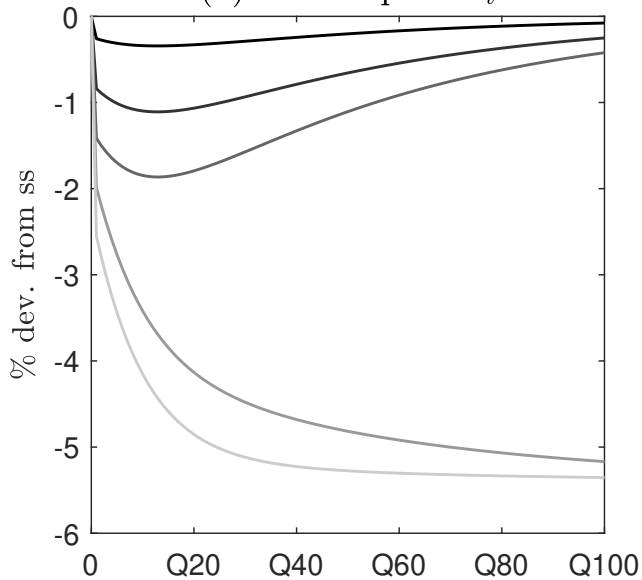

$\varepsilon_{t}^{z}$ takes value between -0.01 and -0.1 . The initial regime $s_{0}=1$. In Panel (b), red dashed line: output threshold below which regime switches from $s_{t-1}=1$ to $s_{t}=2$. Blue dotted line: output threshold above which regime switches from $s_{t-1}=2$ to $s_{t}=1$. Panels (a) and (b): IRF in level. Panels (c) and (d): IRF in percentage deviation from long-run value.

\section{Alternative specifications}

In this section we study alternative specifications of the solution method, the RS rule and the number of regimes. 


\subsection{Alternative projection methods}

The GSSA offers an accurate solution to our model characterized by RS. However, there are alternative specification to the projection method based on fixed grid ${ }^{6}$. We consider two general specifications of the fixed grid method:

\section{Complete bases Chebyshev polynomial}

\section{Smolyak's Polynomial}

In the complete base approach, polynomials of the state variables are built to approximate the decision rules. The polynomials are then projected on a multidimensional grid that covers the ergodic distribution of the state variables. Like for the complete base, the Smolyak method imposes to work with a grid that is held constant throughout the algorithm. The major difference lies in the use of sparse grid in which the number of grid points does not grow exponentially with the number of state variable. Some points of the multidimensional grid are removed as well as some basis function from the polynomials.

Figure 7 shows the fix grids used to compute the solution against the simulation points involved by these method. The fixed grid covers a large area of the state space. As mentioned previously, the ergodic set resembles an ellipsoid, involving that several nodes -especially those located at the cornersare away from the simulated points.

${ }^{6}$ The labeling of the different methods may be sometimes confusing in the literature. In fact, the term "projection method" could apply to the stochastic simulations since it consists in the projection of a polynomial as well. In the original definition of the projection method, labeled "minimum weighted residual method", the grid of the state variables is fixed throughout the algorithm and integrals are evaluated with deterministic integration techniques (quadratures-style). In stochastic simulations the grid is endogenous (simulated points) and Monte Carlo techniques are used for integration by mean of OLS regression. 


\section{Figure 7: Grid coverage}
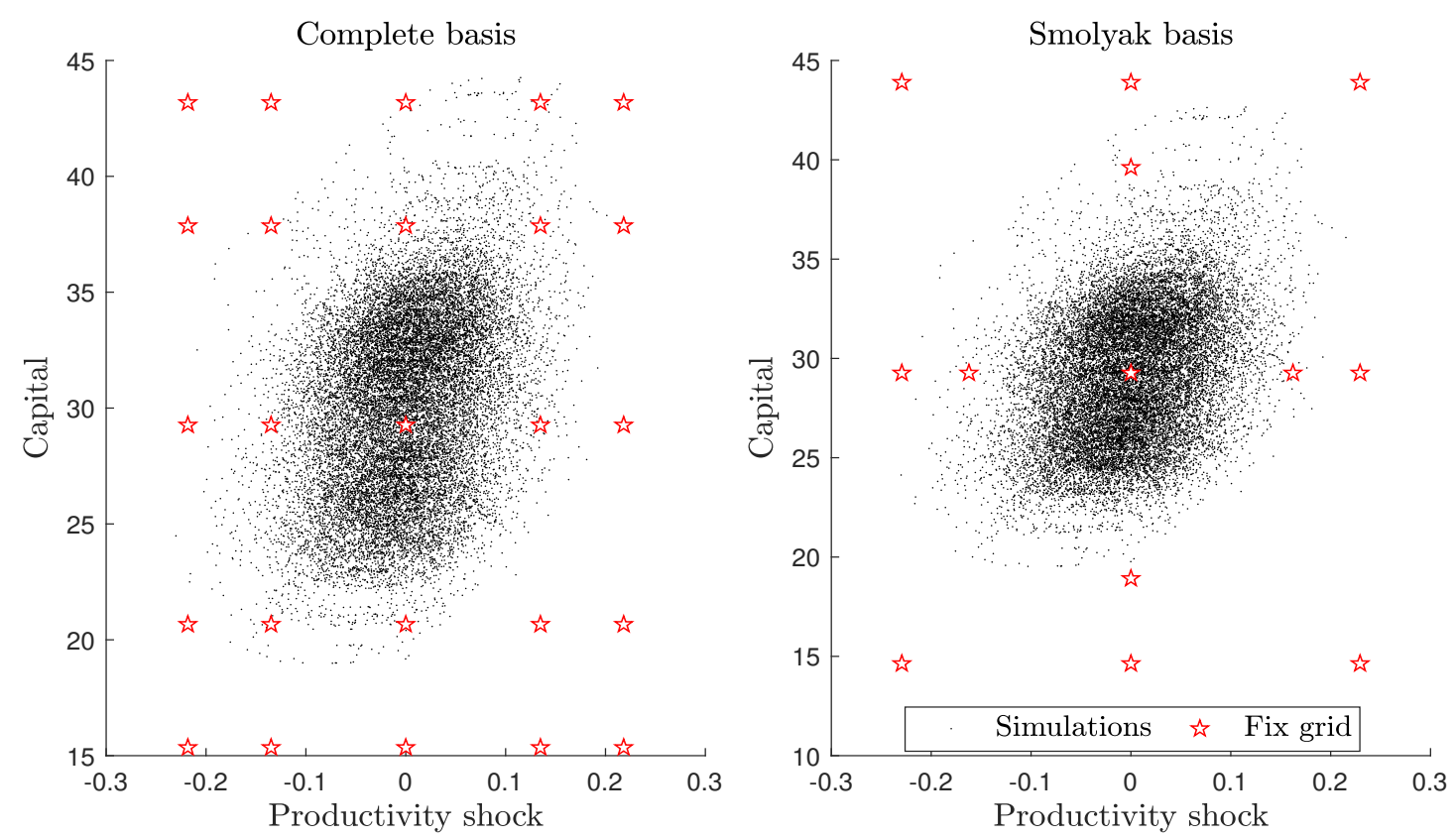

Simulations (black points) are obtained after solving the model with fix grid methods (red marker stars). Chebyshev polynomials in the complete basis are of order 4, as for the GSSA. For Smolyak, $q=4$.

Figure 8 compares the speed and accuracy of the different implementation of the projection method. From an accuracy perspective, the GSSA beats the fixed grid method in our example. The less accurate is the Smolyak's algorithm with maximum EEE well above -3. The complete base Chebyshev polynomial does a decent job in this dimension but remains not as precise as the GSSA.

From a computational time perspective, grid methods are extremely fast compared to the GSSA. The time needed to find the policy rule coefficients is around 26 to 60 times lower for the complete base and the Smolyak's algorithm. The reason is that in this small model with two state variable, the number of nodes in the fixed grid is lower than that of simulated points, even after applying the EDS algorithm. Consequently, the time necessary to evaluate the policy rule is larger in the GSSA than in the fixed grid. However, the grid can increase exponentially with the number of state variables while this is not necessarily the case with the GSSA. In addition, fixed grid compute solution on area of the state space that are never visited in equilibrium. The 
relative good accuracy of the GSSA specification compared to the fixed grid methods comes from the evaluation of the polynomial coefficients on the relevant domain. The points belong to a particular regime for which a policy rule is associated to. In each regime the policy rule is evaluated only on the set of representative points of the corresponding regime, making the precision extremely good. In contrast, in the fixed grid methods, each policy rule is evaluated on the same initial grid. In addition, the grid is extremely large to cover the wide ergodic set that results from the large shock considered in the model. As a consequence, only few grid points cover the area of a given regime that is actually visited in equilibrium. We argue that for small models, up to 5 or 6 state variables, fixed grid methods can be a better choice if the model is not strongly non-linear ${ }^{7}$.

Overall, fix grid methods, albeit fast in our small RBC framework, produce higher EER. Our major recommendation is then to use the GSSA specification for this class of model. However, it would be unfair to reject the fix grid methods on the grounds that they produce larger approximation errors since they do not benefits from the same improvement as the GSSA. For instance, Judd et al. (2014) propose an adaptive Smolyak sparse grid based on stochastic simulations. While they show that it enhances the performance of the Smolyak method fairly well, we rather see it as a refinement of the GSSA since it nests on stochastic simulations and still fits into the representative points scheme. In addition, such a method prevents the use of regime-clustered points as it relies on the minimal number of points consistent with the polynomial basis. It is then impossible to split the grid and to allocate the pieces to the appropriate regime. An alternative could be to use as many adaptive Smolyak sparse grids as there are regime. Each grid would cover the representative points of a particular regime and not the entire ergodic set. Brumm \& Scheidegger (2017) also propose an adaptive sparse grid algorithm but use hierarchical basis functions. They show that their method is accurate and fully parallel which involves significant computational gains. While these option may be a valuable candidate for TBRS, it is beyond the scope of this paper and we leave their implementation open for future research.

\footnotetext{
${ }^{7}$ We also obtain this result with different order of polynomial and different number of quadratures nodes.
} 
Figure 8: Euler Residuals

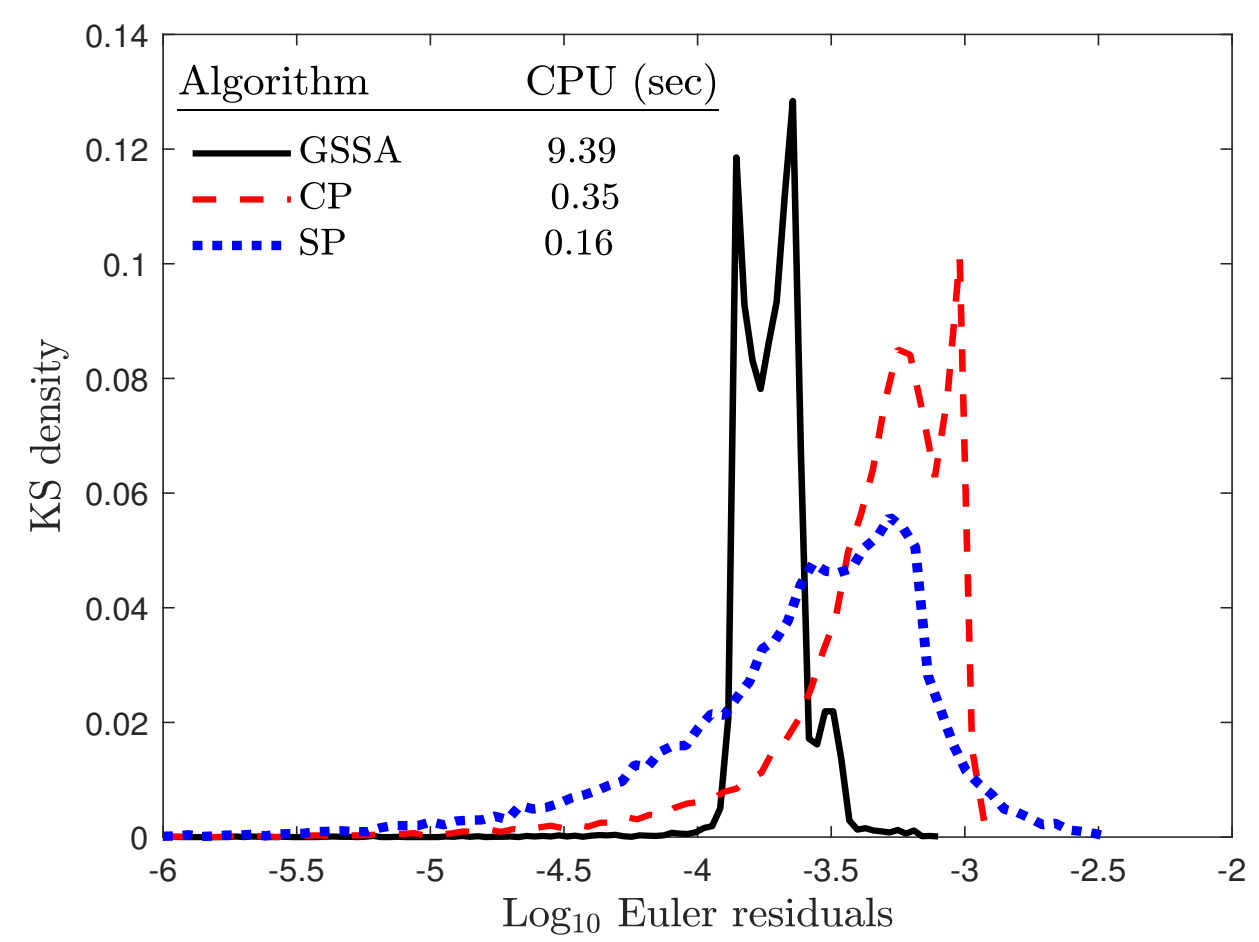

GSSA: Generalized Stochastic Simulation Algorithm. CP: Complete basis polynomial. SP: Smolyak Polynomial. Residuals are computed on each of the 20000 observations (No EDS) from a stochastic simulation with draw on $\varepsilon_{t}^{z}$. Density is displayed. CPU time (in second) for finding the solution.

\subsection{Extension: Stochastic regime switch}

The model studied so far implies that the economy switches regime at a given threshold. The fiscal authorities have full commitment to change the regime when the threshold is reached. There are no possible deviation from the rule set by the authorities. One might assume that the threshold-based government spending rule may be uncertain and this uncertainty may affect agents' expectations. We consider that the regime switching rule now writes:

$$
\begin{aligned}
& s_{t}=\left\{\begin{array}{lll}
1 & \text { if } \quad y_{t}>\underline{y}\left(s_{t-1}\right) \exp \left(x_{t}\right) \\
2 & \text { if } \quad y_{t} \leq \underline{y}\left(s_{t-1}\right) \exp \left(x_{t}\right)
\end{array}\right. \\
& x_{t}=\rho_{x} x_{t-1}+\varepsilon_{t}^{x} \quad \text { with } \quad \varepsilon_{t}^{x} \sim \mathcal{N}\left(0, \sigma_{x}^{2}\right)
\end{aligned}
$$

We refer to $x_{t}$ as an uncertainty shock to the threshold level. The higher 
the volatility of the shock the higher the uncertainty surrounding the regime switch. We set $\rho_{x}$ to 0.9 and $\sigma_{x}$ to 0.1 We solve the model with the GSSA and study the impulse response functions.

Impulse response function. We consider a shock to the threshold that does not cause a RS. This case illustrate pure expectation effects. We assume that the economy is initially $\left(s_{0}\right)$ in regime 1 or 2 . Results are displayed in Figure 9. When $s_{0}=1$, a positive shock $x_{t}$ reduces the distance between $y_{t}$ and $\underline{y}_{t}\left(s_{t-1}\right)$ which increases the probability of switch to regime 2 . This effect crowds out consumption immediately, even if the regime does not change. It leads to more saving, investment and increases the capital stock. This, in turn, raises output. When $s_{0}=2$ a positive shock to $x_{t}$ increases the distance between $y_{t}$ and $y_{t}\left(s_{t-1}\right)$ which lowers the chances of moving to regime 1 in which government spending are lower $g_{t}(1)<g_{t}(2)$. Similarly, such a shock leads to a crowd out in consumption immediately, increase capital and output. 
Figure 9: Impulse response functions

(a) RS shock $x_{t}$

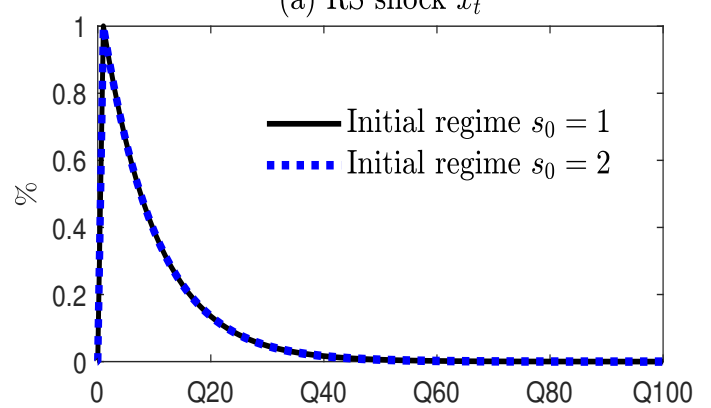

(c) Capital $k_{t}$

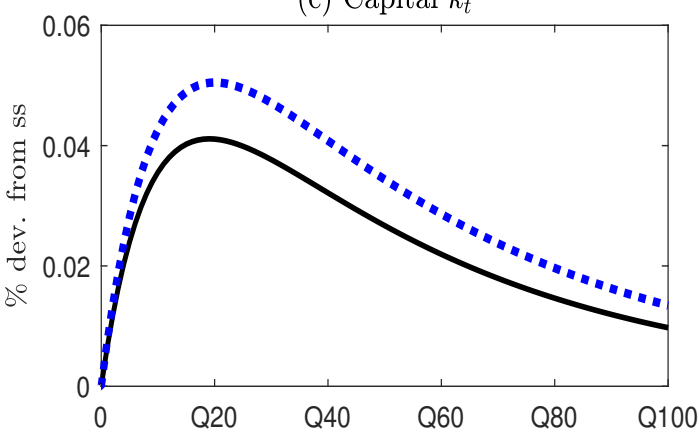

(e) Output threshold $y\left(s_{t}\right)$

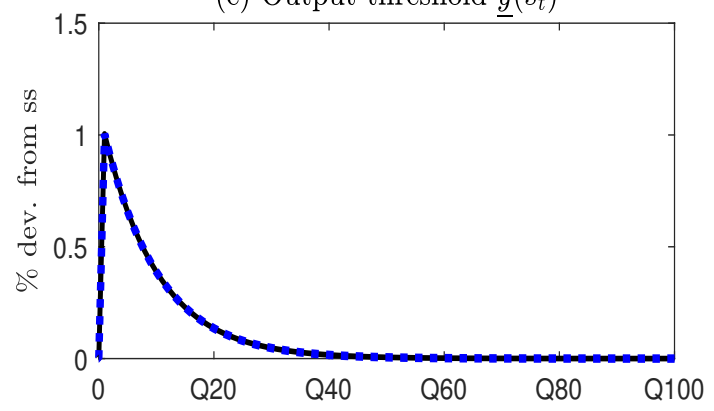

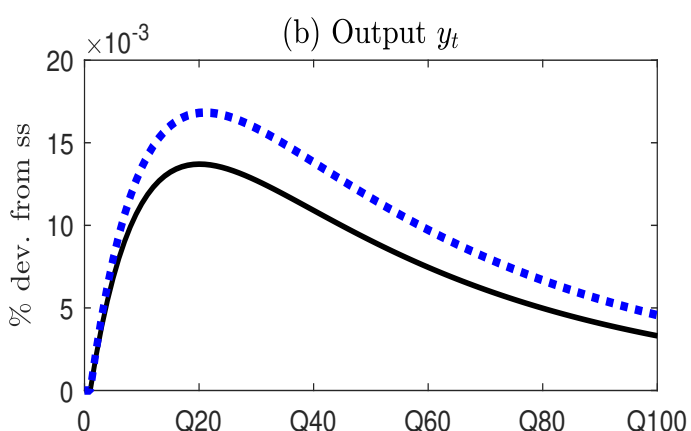

(d) Consumption $c_{t}$

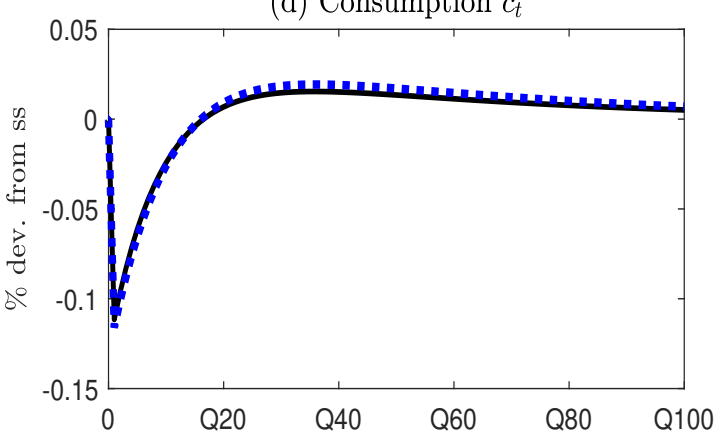

(f) Regime $s_{t}$

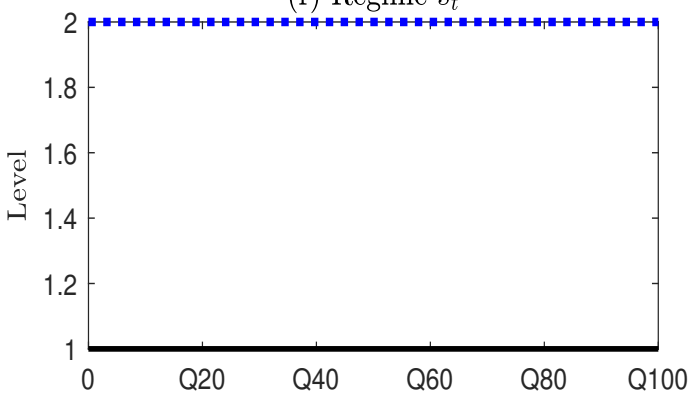

The size of the shock $\varepsilon_{t}^{x}$ is $1 \%$. The initial regime is $s_{0}=1$ (black solid line) or $s_{0}=2$ (blue dashed line). The uncertainty $R S$ shock does not cause a regime switch.

\subsection{More regimes}

We extend the model to allow for additional regimes. The basic RBC model naturally encompasses a regime for investment. When the productivity shock is highly volatile, investment can hit the zero constraint, also known as "irreversible investment". It is a symmetric regime in the sense that the threshold 
value that governs the entry in the zero-investment regime is the same as the one governing the exit.

The extended model consists in introducing in the program (14) the following constraint on investment:

$$
k_{t}-(1-\delta) k_{t-1} \geq 0
$$

The first-order conditions become:

$$
\begin{aligned}
\lambda_{t} & =\left(c_{t}+\rho g_{t}\left(s_{t}\right)\right)^{-\sigma} \\
\lambda_{t}-\mu_{t} & =\beta E_{t}\left[\lambda_{t+1}\left(\alpha \exp \left(z_{t+1}\right) k_{t}^{\alpha-1}+1-\delta\right)-\mu_{t+1}(1-\delta)\right] \\
0 & =\left(k_{t}-(1-\delta) k_{t-1}\right) \mu_{t}
\end{aligned}
$$

where $\mu_{t}$ is the Lagrange multiplier associated to the positive investment constraint 32. There are now four potential regimes. The law of motion for the regime writes:

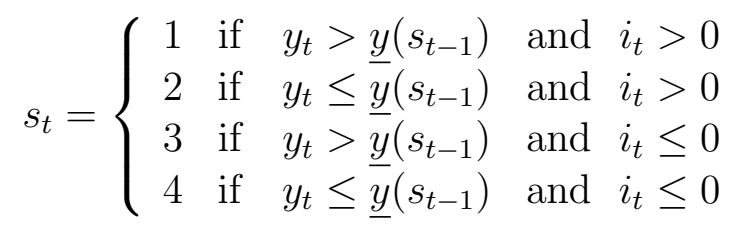

We solve the model with the GSSA and compare the different models in term of accuracy and speed ${ }^{8}$. Figure 10 shows that the accuracy of the solution method remains fairly good. The distribution of the EEE rarely exceed -3 with an average below -4 in all models. The irreversible investment case (model B) involves on average lower EEE. The major reason is that the regime switch is symmetric, thereby avoiding discontinuities in the policy rules. Consequently, the expectations are smoother than in model A and B, which makes it more easy to approximate the solution. When the two types of regime are combined (model $\mathrm{C}$ ) the accuracy is lower and the computational time may increase substantially.

\footnotetext{
${ }^{8}$ Annexe $\mathrm{C}$ of the supplementary appendix provides further information regarding the algorithm and the solution implementation.
} 
Figure 10: Euler residuals

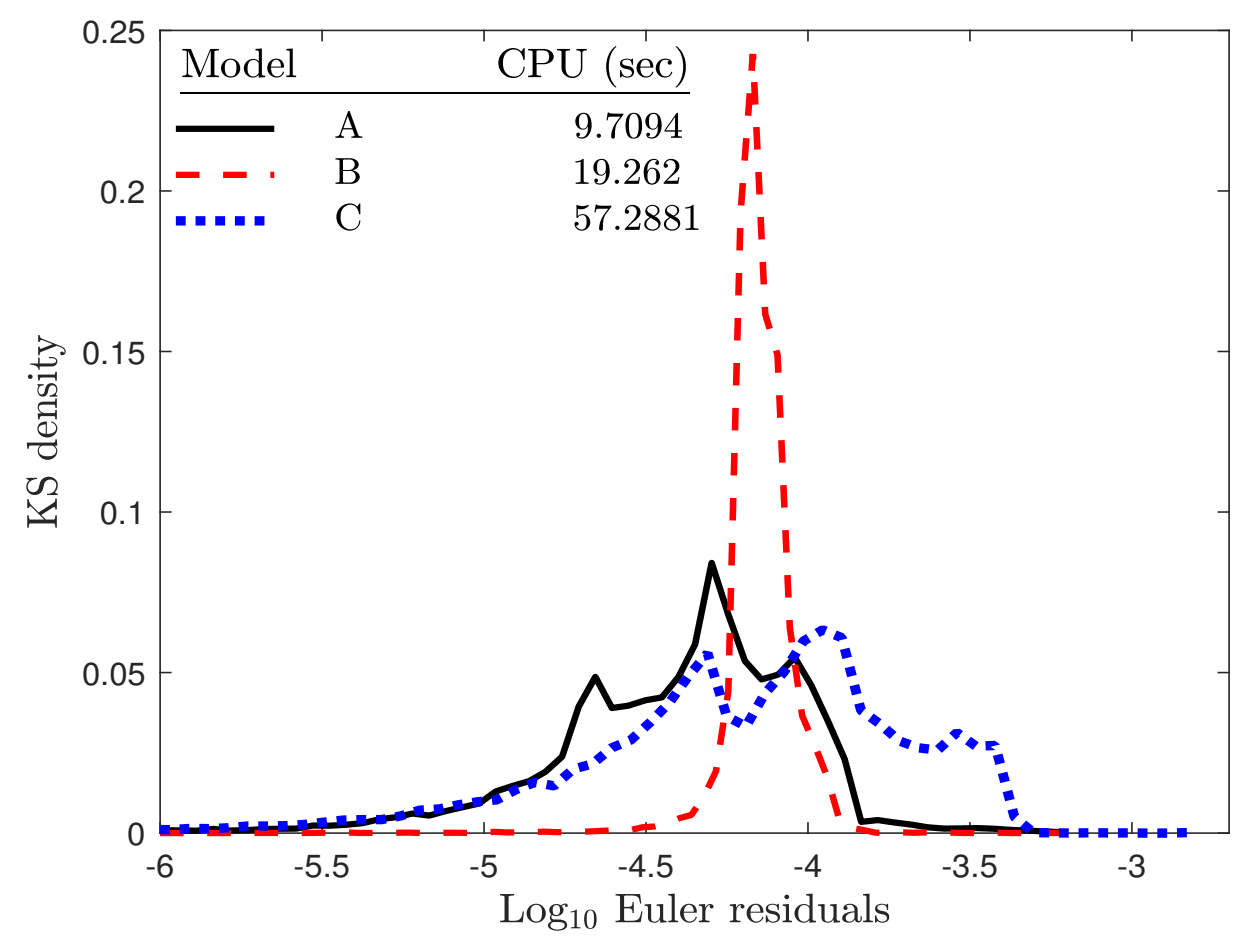

$L_{1}$ mean Euler residuals. $L_{\text {inf }}$ maximum Euler residuals. Residuals are computed on each of the 20000 observations (No EDS) from a stochastic simulation with draw on $\varepsilon_{t}^{z}$. Density estimate is displayed. Model A: benchmark model, two asymmetric regimes based on government spending. Model B: Irreversible investment, two symmetric regimes based on the investment constraint. Model C: Benchmark + Irreversible investment, four potential regimes

\section{Conclusion}

In this paper we introduce TBRS in the canonical RBC framework. We propose a general representation of the RS that encompasses occasionally binding constraints (symmetric RS) and regime-dependent parameters. Our modeling of RS contrasts with existing papers that only consider smooth functions or smooth transition probabilities. In our setup, RS are thresholdbased and may be asymmetric i.e. the thresholds are themselves regimedependent. This class of model is a promising avenue for future research due to their ability to produce infrequent events, persistent regimes and strong non-linearities. Indeed, an important contribution of regime-switching models is that temporary shocks may have very persistent effects. The solution 
algorithm is also well suited for solving model with uncertainty in the regime switching.

Solving this class of model is challenging due to the existence of the aforementioned non-linearities and the discontinuity in the policy rules. To account for RS, we develop an efficient projection method. Our strategy is to consider a specific policy rule associated to each regime and to use a piecewise Chebyshev interpolation. The novelty of the solution method lies in the evaluation of the regime-dependent policy rules in the GSSA approach. Through stochastic simulations, the policy rules are evaluated on an adaptive grid only on the relevant domain: regime-clustered points. This relevant domain is not only a set of representative points but is specific to the regime. In turn, each policy rule is evaluated on an area of the state space that belongs to the corresponding regime, thereby avoiding burdensome and unnecessary calculations. The GSSA produces an accurate and fast solution in all different specifications of the TBRS models which include, kinks, discontinuities and large shocks.

Throughout the paper we solely focus on a very stylized RBC model. The TBRS would fit perfectly into the New Keynesian DSGE models with zero lower bound on the nominal interest rate or with occasional binding financial constraints. Introducing TBRS in medium-to-large scale DSGE models is certainly an important challenge to undertake, especially in terms of computational time. Nonetheless, in a simple example Maliar \& Maliar (2015) already demonstrated the powerfulness of the GSSA in a model of high dimensionality (hundreds of state variables). Furthermore, parallel computing is getting more and more popular in economics (Fernandez-Villaverde \& Valencia (2018)). Another direction in which the solution method can be extended for is parallel computing.

\section{References}

Aldrich, E. M. (2014). Chapter 10 - GPU Computing in Economics. In K. Schmedders \& K. L. Judd (Eds.), Handbook of Computational Economics, volume 3 of Handbook of Computational Economics Vol. 3 (pp. 557-598). Elsevier.

Barthelmann, V., Novak, E., \& Ritter, K. (2000). High dimensional polynomial interpolation on sparse grids. Advances in Computational Mathematics, 12(4), 273-288.

Barthélemy, J. \& Marx, M. (2017). Solving endogenous regime switching models. Journal of Economic Dynamics and Control, 77, 1-25. 
Barthélemy, J. \& Marx, M. (2019). Monetary policy switching and indeterminacy. Quantitative Economics, 10(1), 353-385. Number: 1.

Benigno, G., Foerster, A., Otrok, C., \& Rebucci, A. (2020). Estimating Macroeconomic Models of Financial Crises: An Endogenous RegimeSwitching Approach. Technical Report 26935, National Bureau of Economic Research, Inc.

Bi, H., Leeper, E. M., \& Leith, C. (2013). Uncertain Fiscal Consolidations. The Economic Journal, 123(566), F31-F63.

Bianchi, F. (2013). Regime Switches, Agents' Beliefs, and Post-World War II U.S. Macroeconomic Dynamics. The Review of Economic Studies, 80(2), 463-490. Publisher: Oxford Academic.

Binning, A. \& Maih, J. (2017). Modelling Occasionally Binding Constraints Using Regime-Switching. Technical Report 2017/23, Norges Bank.

Boneva, L., Harrison, R., \& Waldron, M. (2018). Threshold-based forward guidance. Journal of Economic Dynamics and Control, 90, 138-155.

Brumm, J. \& Scheidegger, S. (2017). Using Adaptive Sparse Grids to Solve High-Dimensional Dynamic Models. Econometrica, 85(5), 1575-1612.

Canova, F., Ferroni, F., \& Matthes, C. (2020). Detecting and Analyzing the Effects of Time-Varying Parameters in Dsge Models. International Economic Review, 61(1), 105-125.

Chang, Y., Choi, Y., \& Park, J. Y. (2017). A new approach to model regime switching. Journal of Econometrics, 196(1), 127 - 143.

Chang, Y., Maih, J., \& Tan, F. (2019). Origins of monetary policy shifts: A new approach to regime switching in dsge models. Norges Bank Working Paper.

Christiano, L. J. \& Fisher, J. D. M. (2000). Algorithms for solving dynamic models with occasionally binding constraints. Journal of Economic Dynamics and Control, 24(8), 1179-1232.

Chung, H., Davig, T., \& Leeper, E. M. (2007). Monetary and Fiscal Policy Switching. Journal of Money, Credit and Banking, 39(4), 809-842. _eprint: https://onlinelibrary.wiley.com/doi/pdf/10.1111/j.15384616.2007.00047.x. 
Davig, T., Leeper, E. M., Clarida, R. H., \& Lindé, J. (2006). Endogenous monetary policy regime change [with comments]. NBER International Seminar on Macroeconomics, (pp. 345-391).

Demkowicz, L. (2006). Computing with hp-ADAPTIVE FINITE ELEMENTS: Volume 1 One and Two Dimensional Elliptic and Maxwell Problems. CRC Press.

Demkowicz, L., Rachowicz, W., \& Devloo, P. (2002). A Fully Automatic hp -Adaptivity. Journal of Scientific Computing, 17(1), 117-142.

Farmer, R. E. A., Waggoner, D. F., \& Zha, T. (2009). Understanding Markov-switching rational expectations models. Journal of Economic Theory, 144(5), 1849-1867.

Fernandez-Villaverde, J., Rubio-Ramirez, J. F., \& Schorfheide, F. (2016). Solution and Estimation Methods for DSGE Models. In Handbook of Macroeconomics, volume 2 (pp. 527-724). Elsevier.

Fernandez-Villaverde, J. \& Valencia, D. Z. (2018). A Practical Guide to Parallelization in Economics. Technical Report 24561, National Bureau of Economic Research, Inc.

Fève, P., Matheron, J., \& Sahuc, J.-G. (2013). A Pitfall with Estimated DSGE-Based Government Spending Multipliers. American Economic Journal: Macroeconomics, 5(4), 141-178.

Halton, J. H. (1964). Algorithm 247: Radical-inverse quasi-random point sequence.

Hamilton, J. D. (1994). Time Series Analysis. Princeton, N.J: Princeton University Press.

Hamilton, J. D. (2016). Chapter 3 - Macroeconomic Regimes and Regime Shifts. In J. B. Taylor \& H. Uhlig (Eds.), Handbook of Macroeconomics, volume 2 (pp. 163-201). Elsevier.

Hirose, Y. \& Sunakawa, T. (2019). Review of solution and estimation methods for nonlinear dynamic stochastic general equilibrium models with the zero lower bound. The Japanese Economic Review, 70(1), 51-104.

Judd, K. L. (1992). Projection methods for solving aggregate growth models. Journal of Economic Theory, 58(2), 410-452. 
Judd, K. L. (1998). Numerical Methods in Economics, volume 1. The MIT Press.

Judd, K. L., Maliar, L., \& Maliar, S. (2011). Numerically stable and accurate stochastic simulation approaches for solving dynamic economic models. Quantitative Economics, 2(2), 173-210.

Judd, K. L., Maliar, L., Maliar, S., \& Valero, R. (2014). Smolyak method for solving dynamic economic models: Lagrange interpolation, anisotropic grid and adaptive domain. Journal of Economic Dynamics and Control, $44,92-123$.

Krueger, D. \& Kubler, F. (2004). Computing equilibrium in OLG models with stochastic production. Journal of Economic Dynamics and Control, 28(7), 1411-1436.

Leeper, E. M. \& Zha, T. (2003). Modest policy interventions. Journal of Monetary Economics, 50(8), 1673-1700.

Liu, Z., Waggoner, D. F., \& Zha, T. (2011). Sources of macroeconomic fluctuations: A regime-switching DSGE approach. Quantitative Economics, 2(2), 251-301. _eprint: https://onlinelibrary.wiley.com/doi/pdf/10.3982/QE71.

Maliar, L. \& Maliar, S. (2015). Merging simulation and projection approaches to solve high dimensional problems with an application to a new Keynesian model. Quantitative Economics, 6(1), 1-47.

Malin, B. A., Krueger, D., \& Kubler, F. (2011). Solving the multi-country real business cycle model using a Smolyak-collocation method. Journal of Economic Dynamics and Control, 35(2), 229-239.

Nakata, T. (2017). Uncertainty at the Zero Lower Bound. American Economic Journal: Macroeconomics, 9(3), 186-221.

Patera, A. T. (1984). A spectral element method for fluid dynamics: Laminar flow in a channel expansion. Journal of Computational Physics, 54(3), 468488.

Sims, C. A. \& Zha, T. (2006). Were There Regime Switches in U.S. Monetary Policy? American Economic Review, 96(1), 54-81.

Smolyak, S. (1963). Quadrature and interpolation formulas for tensor products of certain classes of functions. 
Svensson, L. E. O. \& Williams, N. (2009). Optimal Monetary Policy under Uncertainty in DSGE Models: A Markov Jump-Linear-Quadratic Approach. In Central Banking, Analysis, and Economic Policies Book Series, volume 13 (pp. 077-114). Central Bank of Chile.

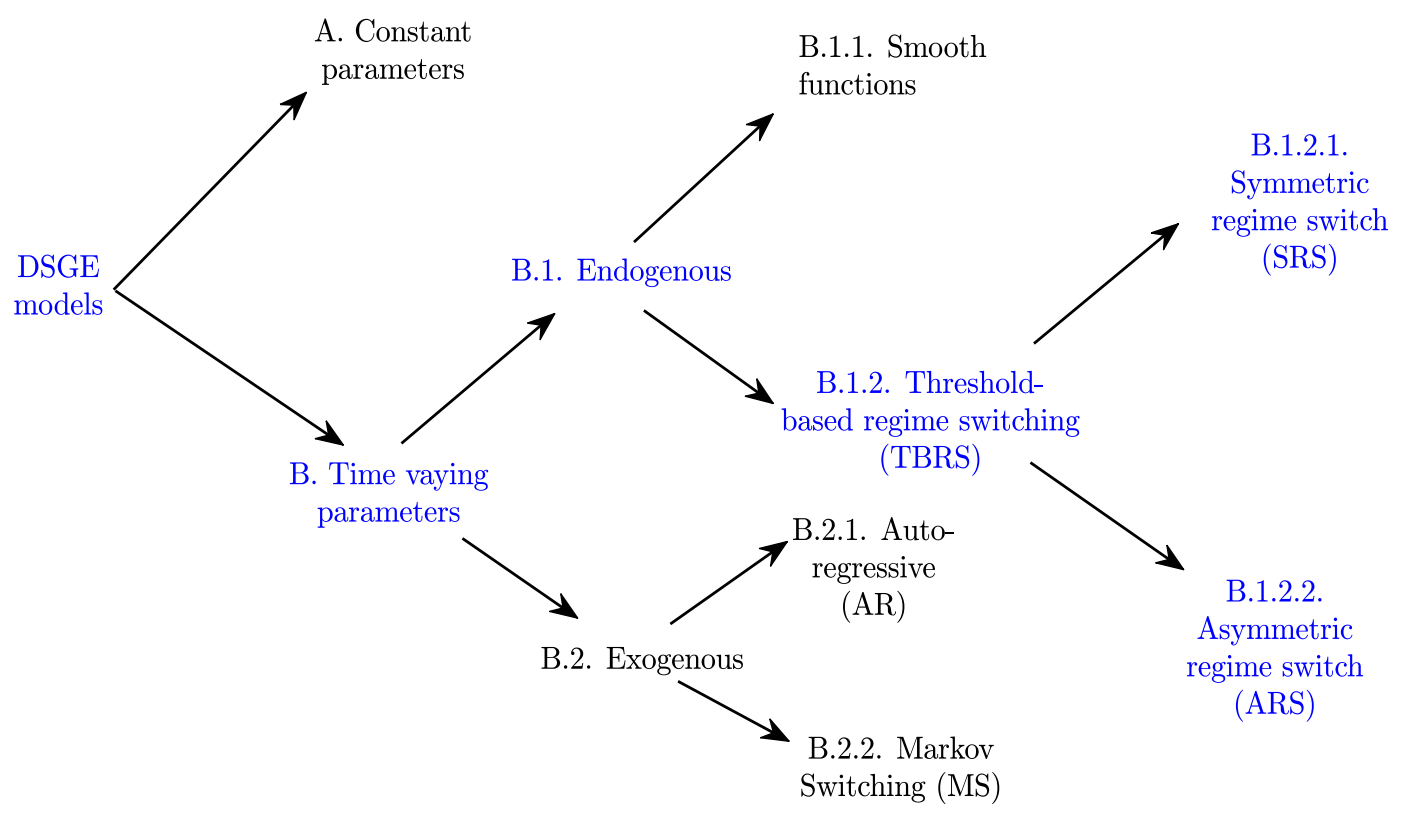

Figure 11: Regime change category . 


\section{Online Appendix (not for publication)}

\section{A Hamilton's representation of RS}

We provide an alternative formulation of the RS. We follow Hamilton (1994, 2016) and adopt a relatively standard exposition that encompasses the popular MS case as well as the symmetric RS case. The major difference is the use of a matrix representation for the law of motion of the regime. Denote by $\boldsymbol{\xi}_{t}$ a $[N \times 1]$ vector summarizing the regime at date $t$ whose $i$ th element is unity when $s_{t}=i$ and is zero otherwise:

$$
\boldsymbol{\xi}_{t}=\left[\begin{array}{c}
\mathbb{1}_{\left\{s_{t}=1\right\}} \\
\mathbb{1}_{\left\{s_{t}=2\right\}} \\
\vdots \\
\mathbb{1}_{\left\{s_{t}=N\right\}}
\end{array}\right]
$$

As shown in Hamilton (1994), the vector $\boldsymbol{\xi}_{t}$ has the following AR(1) representation:

$$
\xi_{t}=Q \xi_{t-1}+u_{t}
$$

$u_{t}$ is a $(N \times 1)$ vector corresponding to a discrete-valued martingale difference sequence whose elements always sum to zero, that is $E_{t-1} u_{t}=0 . Q$ is a $(N \times N)$ transition matrix:

$$
Q=\left[\begin{array}{cccc}
q_{11} & q_{21} & \ldots & q_{N 1} \\
q_{12} & q_{22} & \ldots & q_{N 2} \\
\vdots & \vdots & \ddots & \vdots \\
q_{1 N} & q_{2 N} & \ldots & q_{N N}
\end{array}\right]
$$

$q_{i j}$ is the probability that the regime switch to state $i$ when the initial regime $s_{t-1}=j$. When the probabilities $q_{i j}$ are constant, the above representation is a typical MS process. In a TBRS environment, $p_{i j}$ are endogenous and $u_{t}=0 \forall t$. From Equation 36 we still impose that columns of $Q$ sum to unity such that $\boldsymbol{\xi}_{t}$ is a stationary process. The transition probabilities $p_{i j}$ are given by:

$$
q_{i j}=\mathbb{1}_{\left\{v _ { t } \in \left[\underline{v}_{i}(j), \underline{v}_{i+1}(j)[\}\right.\right.}
$$


It is straightforward to see that $\sum_{j} p_{i j}=1$ as $v_{t}$ can not be in two regimes at the same time by definition. With a condition on $L$ variables, one has:

$$
q_{i j}=\prod_{\ell=1}^{L} \mathbb{1}_{\left\{v_{t}^{\ell} \in \underline{v}_{i_{\ell}}^{\ell}(j), \underline{v}_{i_{\ell}+1}^{\ell}(j)[\}\right.}
$$

Finally the parameter value can be written in matrix form as:

$$
p\left(s_{t}\right)=[p(1), p(2), \ldots, p(N)] \boldsymbol{\xi}_{t}
$$

\section{B Solution method}

An overview of the approximation methods in the literature can be found in Fernandez-Villaverde et al. (2016) and Hirose \& Sunakawa (2019). The solution method considered in this paper lies at the intersection of several other method. First, it is a global approximation method. The solution of the dynamic system is evaluated over the ergodic distribution and not only around a deterministic steady state like for local approximation. There are different method of global approximation: value function iteration, time iteration, parameterized expectation algorithm, finite element, projection method, etc. Our algorithm is based on the projection method but shares some similarities with the finite element methods. Indeed, the algorithm consists in estimating coefficients of a set of orthogonal polynomials to approximate the decision rules. However, the grid on which these polynomials are projected is (1) simulated (simulation methods), (2) reduced (with EDS techniques) and (3) partitioned according to the regime. The last aspect belongs to Spectral Element Method (SEM) originally developed by Patera (1984) in fluid mechanics which combines the finite element method and the spectral method. In finite element low-order finite element expansions are considered while the spectral method uses higher order of the approximation in each element or, equivalently more basis functions. In our case the element are determined by the regime switching conditions. They allow to split the state-space and to apply high-order approximation on each sub-domain.

\section{B.1 Some useful notations and operators}

Throughout the algorithm we will use the following notations and operators.

- $\underset{[l \times c]}{x}$ defines $x$ as a matrix with $l$ lines and $c$ columns.

- $\boldsymbol{x}_{t}=\left\{z_{t}, k_{t-1}\right\}$ defines the state vector (excluding the regime). 
- $s_{t}$ defines the regime.

- $n=1, \ldots, N$ denote the regime number.

- $\mathcal{I}_{m}$ is $[m \times 1]$ column vector whose elements are each equal to one.

- $\mathbb{1}_{\{\text {cond }\}}$ is an indicator variable taking the value 1 if the condition is satisfied and zero otherwise.

- $d=1, \ldots, \boldsymbol{D}$ is the approximation order of the Chebyshev polynomial.

- $q$ is the parameter controlling the degree of approximation in the Smolyak method.

- $m=1, \ldots, \boldsymbol{M}$ is the $m$-th nodes for each individual state variable in the fixed grid approach.

- $k=1, \ldots, K$ is the index of continuous state variables.

- $h=1, \ldots, H$ is the $h$-th quadrature nodes and weight used for integration.

- $\otimes$ stands for the Kronecker product.

- $\circ$ stands for the Hadamar product (or Schur product). It is an element by element product.

- $P\left(x_{t} ; \Theta(s)\right)$ the approximation function of the decision rule (control variable) that depends on the vector of state variables $\boldsymbol{x}_{t}$ and a set of regimedependent polynomial coefficients $\Theta(s)$.

- $\boldsymbol{x}^{\prime}=\Gamma\left(\boldsymbol{x}, \varepsilon^{\prime} ; \Theta(s)\right)$ the law motion for the state variables given that the control variables are approximated by the function $P($.$) .$

\section{B.2 Preliminary results on Chebyshev functions}

In order to approximate the unknown functions (the policy rules) we use Chebyshev polynomials. 


\section{B.2.1 One-dimensional approximation}

Consider first that $\boldsymbol{x}_{t}$ is a one-dimensional state vector. The function $\varphi\left(\boldsymbol{x}_{t}\right)$ ensures that $\boldsymbol{x}_{t}$ fits into the Chebyshev domain $[-1,1]$ :

$$
\varphi\left(x_{t}\right)=2 \frac{x_{t}-a}{b-a}-1
$$

Chebyshev polynomial basis of order $d \in[2, \boldsymbol{D}]$ are built according to the following recursion:

$$
T_{d+1}\left(\boldsymbol{S}_{t}\right)=\boldsymbol{x}_{t} T_{d}\left(\boldsymbol{x}_{t}\right)-T_{d-1}\left(\boldsymbol{x}_{t}\right)
$$

with $T_{0}\left(\boldsymbol{x}_{t}\right)=1$ and $T_{1}\left(\boldsymbol{x}_{t}\right)=\boldsymbol{x}_{t}$. Applying the trigonometric identities, the $\mathrm{d}$-th member of the polynomial is:

$$
T_{d}\left(\boldsymbol{x}_{t}\right)=\cos \left(d \arccos \left(\boldsymbol{x}_{t}\right)\right)
$$

Let $P($.$) be an approximation function of a one-dimensional state variable$ $\boldsymbol{x}_{t}$. It writes:

$$
P\left(\boldsymbol{x}_{t} ; \Theta\right)=\sum_{d=0}^{D} \theta_{d} T_{d}\left(\varphi\left(\boldsymbol{x}_{t}\right)\right)
$$

where $\Theta=\left\{\theta_{1}, \ldots, \theta_{\boldsymbol{D}}\right\}$ are the parameters of the Chebyshev function.

\section{B.2.2 Multidimensional approximation}

When the number of state variables is higher than one $(K>1)$, we have to build a multidimensional Chebyshev polynomial basis. The $K$-fold tensor product basis for the function with $K$ variables $\left(\boldsymbol{x}_{t}=\left\{x_{t}^{1}, \ldots, x_{t}^{K}\right\}\right)$ is built by taking all possible $K$-term products of $T_{d_{k}}$. The tensor product basis $\mathcal{B}^{T}$ is defined as:

$$
\underset{\left[1 \times(1+d \boldsymbol{K})^{d}\right]}{\mathcal{B}^{T}}=\left\{\prod_{k=1}^{\boldsymbol{K}} T_{d_{k}}\left(\varphi\left(x_{t}^{k}\right)\right) \mid d_{k}=0, \ldots, \boldsymbol{D} ; k=1, \ldots, \boldsymbol{K}\right\}
$$

The problem of the tensor product basis is that some of the basis have an order higher than $\boldsymbol{D}$ which increase the number of elements. In the complete base approach, all products of polynomial terms must have an order not higher than $D$. Denote by $\mathcal{B}^{C}$ the complete polynomial basis with $\mathcal{B}^{C} \subseteq \mathcal{B}^{T}$. It is given by:

$$
\begin{aligned}
\underset{\left.\mathcal{B}^{C} \times \boldsymbol{b}\right]}{\mathcal{B}^{C}=} & \left\{\prod_{k=1}^{\boldsymbol{K}} T_{d_{k}}\left(\varphi\left(x_{t}^{k}\right)\right) \mid \sum_{d_{1}=0}^{\boldsymbol{D}} \ldots \sum_{d_{\boldsymbol{K}}=0}^{\boldsymbol{D}}\left(\sum_{j=1}^{\boldsymbol{K}} d_{j}\right) \leq \boldsymbol{D}\right\} \\
& d_{k}=0, \ldots, \boldsymbol{D} \\
& k=1, \ldots, \boldsymbol{K}
\end{aligned}
$$


where $\boldsymbol{b}$ is the number polynomial basis such that:

$$
\boldsymbol{b}=\sum_{d_{1}=0}^{\boldsymbol{D}} \ldots \sum_{d_{\boldsymbol{K}}=0}^{\boldsymbol{D}} \mathbb{1}_{\left\{\sum_{j=1}^{K} d_{j} \leq \boldsymbol{D}\right\}} \quad \text { for } \quad k=1, \ldots, \boldsymbol{K}
$$

The multidimensional approximation function writes:

$$
P\left(x_{t} ; \Theta\right)=\mathcal{B}^{C} \circ \Theta
$$

where $\Theta=\left\{\theta_{1}, \ldots, \theta_{\boldsymbol{b}}\right\}^{\top}$ is a $[\boldsymbol{b} \times 1]$ vector of Chebyshev coefficients.

\section{B.2.3 Piecewise approximation}

In the algorithm described latter we use piecewise approximation. The general idea is that the decision rule (consumption) is approximated by a collection of approximation functions, each of them belongs to a particular regime. Consequently, they are as many polynomials as there are regimes ${ }^{9}$. We adopt simplified notations. We denote by $P\left(\boldsymbol{x}_{t} ; \Theta(s)\right)$ the approximation function of the control variable when the regime is $s$ with $\Theta(s)=\left\{\theta_{1}(s), \ldots, \theta_{m}(s)\right\}^{\top}$ the vector of coefficients that belongs to the regime $s$. This notation mean that the decisions rules, summarized by the coefficient $\Theta(s)$, are actually time-varying. Since the regime $s$ is a dummy state variable, it can not be treated as capital or the productivity shock. Therefore it is excluded from the state vector $\boldsymbol{x}_{t}$. The "Aggregate" policy rule is defined as:

$$
P\left(\boldsymbol{S}_{t} ; \Theta\right)=\left\{\begin{array}{cccc}
P\left(\boldsymbol{x}_{t} ; \Theta(1)\right) & \text { if } & s_{t}=1 \\
P\left(\boldsymbol{x}_{t} ; \Theta(2)\right) & \text { if } & s_{t}=2 \\
\vdots & & \vdots \\
P\left(\boldsymbol{x}_{t} ; \Theta(N)\right) & \text { if } & s_{t}=N
\end{array}\right.
$$

with $\Theta=\{\Theta(1), \ldots, \Theta(N)\}$ being a set of Chebyshev coefficients.

\section{B.3 Grid of the state variables with complete basis}

For the sake of clarity subscripts will correspond to the nodes of the grid in place of the time. The grid defines the state-space over which the Chebyshev polynomials will be projected. Because the number of state variables is higher

\footnotetext{
${ }^{9}$ This method approximates the solution more accurately than if we use a single Chebyshev polynomial. It allows to manage the kink or RS in the policy rules. It is important to note that the two policy rules are linked when the expectations are computed. It means that agents take into account the endogenous RS process when they take their decisions.
} 
than one, we need to construct a multidimensional grid. Under the complete product basis the number of knots for each state variable must be equal or higher than the order of the polynomial plus one: $\boldsymbol{M} \geq \boldsymbol{D}+1$. We simply assume that $\boldsymbol{M}=\boldsymbol{D}+1$. We use the extrema of Chebychev polynomials as the basis for the grid points. $\mathcal{R}^{M}=\left\{r_{1}, \ldots, r_{M}\right\} \subset[-1,1]$ is the set of the extrema of the Chebychev polynomials knots. The roots of the Chebyshev polynomial are given by:

$$
r_{m}=-\cos \left(\frac{2 m-1}{2 \boldsymbol{M}} \pi\right) \text { for } \quad m=1, \ldots, \boldsymbol{M}
$$

The grid for each state variable $k$ at each node $n$ is defined by:

$$
\boldsymbol{g}_{m}^{k}=x_{\min }^{k}+\left(r_{m}+1\right) \frac{x_{\max }^{k}-x_{\min }^{k}}{2} \text { for } m=1, \ldots, \boldsymbol{M} ; \quad k=1, \ldots, \boldsymbol{K}
$$

where $x_{\min }^{k}$ and $x_{\max }^{k}$ denote the lower and the upper bound of the domain of the state variable $x^{k}$ respectively. The multidimensional grid $\mathcal{G}$ is obtained through a tensor product over each individual grid:

$$
\underset{\left[M^{K} \times K\right]}{\mathcal{G}}=\left\{\mathcal{G}^{1}, \ldots, \mathcal{G}^{K}\right\}
$$

where,

$$
\underset{\left[\boldsymbol{M}^{\boldsymbol{K}} \times 1\right]}{\mathcal{C}^{k}}=\underset{\left[\boldsymbol{M}^{k-1} \times 1\right]}{\mathcal{I}_{\boldsymbol{M}^{k-1}}} \otimes \underset{[\boldsymbol{M} \times 1]}{\varphi\left(\boldsymbol{g}^{k}\right)} \otimes \underset{\left[\boldsymbol{M}^{\boldsymbol{K}-k} \times 1\right]}{\mathcal{I}_{\boldsymbol{K}-k}}
$$

Policy rule approximations Let $P(\mathcal{G} ; \Theta(s))$ be the policy rule of a control variable when the regime is $s$ projected on the multidimensional grid $\mathcal{G}$. As before, $\Theta(s)$ stands for the vector of coefficients of the approximation function in regime $s$. The approximation function writes:

$$
P(\mathcal{G} ; \Theta(s))=\mathcal{B}^{C}(\mathcal{G}) \circ \Theta(s)
$$

\section{B.4 The Smolyak method}

\section{B.4.1 Overview}

Like the complete basis, the Smolyak method belongs to fixed grid methods. The major difference is that in the Smolyak method the grid is sparse and several polynomial basis are eliminated. We summarize below how the Smolyak method works and advise the readers to refer to Smolyak (1963), Barthelmann et al. (2000), Krueger \& Kubler (2004), Malin et al. (2011) and Judd et al. (2014) for an implementation of the method in macroeconomics. 
The ideas is to build a sparse grid of points covering the ergodic distribution of the variable. This grid labeled by $\mathcal{G}(q, K)$ is a function of the number of state variables and a parameter $q$ controlling for the size of the grid and the approximation level. Let $P(\mathcal{G} ; \Theta(s))$ be an approximation function. The idea behind the Smolyak method is that for each grid point $\boldsymbol{g}_{m}, m=1, \ldots, \boldsymbol{M}$, $\boldsymbol{g}_{m} \in \mathcal{G}(q, \boldsymbol{K})$ we have:

$$
g\left(\boldsymbol{g}_{m} ; p(s)\right)=P\left(\boldsymbol{g}_{m} ; \Theta(s)\right)
$$

where $g($.$) is the "true" policy rule but is unknown. At the grid point of$ $\mathcal{G}(q, \boldsymbol{K})$ the approximation is exact, outside it is close.

\section{B.4.2 Grid}

Let first investigate the built of the grid. As before the grid encompasses as many elements as we have state variables: $\left\{\mathcal{G}^{1}, \ldots, \mathcal{G}^{K}\right\}$ where each element is a set of Clenshaw-Curtis points that are based on the extrema of the Chebyshev polynomials:

$$
\mathcal{G}^{k}=\left\{\boldsymbol{g}_{1}^{k}, \ldots, \boldsymbol{g}_{m_{k}}^{k}\right\}
$$

and

$$
\boldsymbol{g}_{j}^{k}=-\cos \left(\frac{j-1}{m_{k}}\right), \quad j=1, \ldots, m_{k}
$$

The sparse grid is calculated as the cartesian product of individual grid:

$$
\mathcal{G}(q, \boldsymbol{K})=\bigcup_{q-\boldsymbol{K}+1 \leq|i| \leq q}\left(\mathcal{G}^{i_{1}} \times \ldots \times \mathcal{G}^{i_{\boldsymbol{K}}}\right)
$$

where

$$
|i|=\sum_{k}^{K} i_{k}
$$

\section{B.4.3 Function approximation}

The Smolyak method consists in interpolating a polynomial function on the grid $\mathcal{G}(q, \boldsymbol{K})$. Denote by $\tilde{P}^{|i|}(\boldsymbol{x} ; \Theta(s))=\mathcal{B}^{T}(\boldsymbol{x}) \circ \Theta(s)$ the multivariate polynomial with tensor product base (see section A.2.2 for calculation) of degree $|i|$. The Smolyak polynomial, based on the tensor product basis, is defined as follow:

$$
P(\boldsymbol{x} ; \Theta(s), q, \boldsymbol{K})=\sum_{q-\boldsymbol{K}+1 \leq|i| \leq q}(-1)^{q-|i|}\left(\begin{array}{c}
\boldsymbol{K}-1 \\
q-|i|
\end{array}\right) \tilde{P}^{|i|}(\boldsymbol{x} ; \Theta(s))
$$




\section{B.5 Numerical integration}

Euler equations involve two important difficulties: (1) the presence of the aggregate shock and (2) the RS that may occur in the next period. Since the regime changes according to the aggregate shock the evaluation of the expectation functions must take into account potential RS. To simplify notation, we drop time subscript $t$ from present variable and use $x^{\prime}$ to define the next period state variables. Consider the Euler equation :

$$
(P(\boldsymbol{x} ; \Theta(s))+\rho g(s))^{-\sigma}=\beta E \mathcal{E}\left(\boldsymbol{x}^{\prime}, P\left(\boldsymbol{x}^{\prime} ; \Theta\left(s^{\prime}\right)\right)\right)
$$

where $\mathcal{E}($.$) stands for the expectation function and E$ the expectation operator. The solution of Next period state variables are obtained using the following law of motion:

$$
\boldsymbol{x}^{\prime}=\Gamma\left(x, \varepsilon^{\prime} ; \Theta(s)\right)
$$

Note that, contrary to the main paper, here the function $\Gamma($.$) governs the$ evolution of the state vector $\boldsymbol{x}=\left\{k_{t-1}, z_{t}\right\}$, not the evolution of the regime $s$. The next period regime $s$ depends on output which is composed of today's productivity shock and the last period capital stock. We use the following evolution for the regime:

$$
s^{\prime}=\psi\left(s, \boldsymbol{x}^{\prime}\right) \equiv \psi\left(s, \Gamma\left(\boldsymbol{x}, \varepsilon^{\prime} ; \Theta(s)\right)\right)
$$

The Euler equation can then be rewritten as:

$$
(P(\boldsymbol{x} ; \Theta(s))+\rho g(s))^{-\sigma}=\beta \int_{\varepsilon^{\prime}} \mathcal{E}\left(\Gamma\left(\boldsymbol{x}, \varepsilon^{\prime} ; \Theta(s)\right), P\left(\Gamma\left(\boldsymbol{x}, \varepsilon^{\prime} ; \Theta(s)\right) ; \Theta\left(\psi\left(s, \Gamma\left(\boldsymbol{x}, \varepsilon^{\prime} ; \Theta(s)\right)\right)\right)\right)\right)
$$

In order to solve the numerical integration problem, we use Gauss-Hermite quadratures. It consists in evaluating the integral at different nodes and summing the evaluations using particular weights. Let $\boldsymbol{e}_{h}$ be the $h$-th nodes and $\boldsymbol{\omega}_{h}$ the $h$-th weights of the Gauss-Hermite quadratures with $h=1, \ldots, H$, $H$ being the number of quadrature nodes ${ }^{10}$.

Let $\Xi$ be an approximation function of the integral (the right-hand-side of the previous equation). With Gauss-Hermite quadratures, one has:

$\Xi=\frac{\beta}{\sqrt{\pi}} \sum_{h=1}^{H} \boldsymbol{\omega}_{h} \mathcal{E}\left(\Gamma\left(\boldsymbol{x}, \sigma_{z} \sqrt{2} \boldsymbol{e}_{h} ; \Theta(s)\right), P\left(\Gamma\left(\boldsymbol{x}, \sigma_{z} \sqrt{2} \boldsymbol{e}_{h} ; \Theta(s)\right) ; \Theta\left(\psi\left(s, \boldsymbol{x}, \sigma_{z} \sqrt{2} \boldsymbol{e}_{h} ; \Theta(s)\right)\right)\right)\right)$

We then obtain an estimate of the decision rule:

$$
\hat{c}(s)=\Xi^{-\frac{1}{\sigma}}-\rho g(s)
$$

\footnotetext{
${ }^{10}$ With multiple shock, one must use a tensor product.
} 


\section{B.6 Overview of the algorithms}

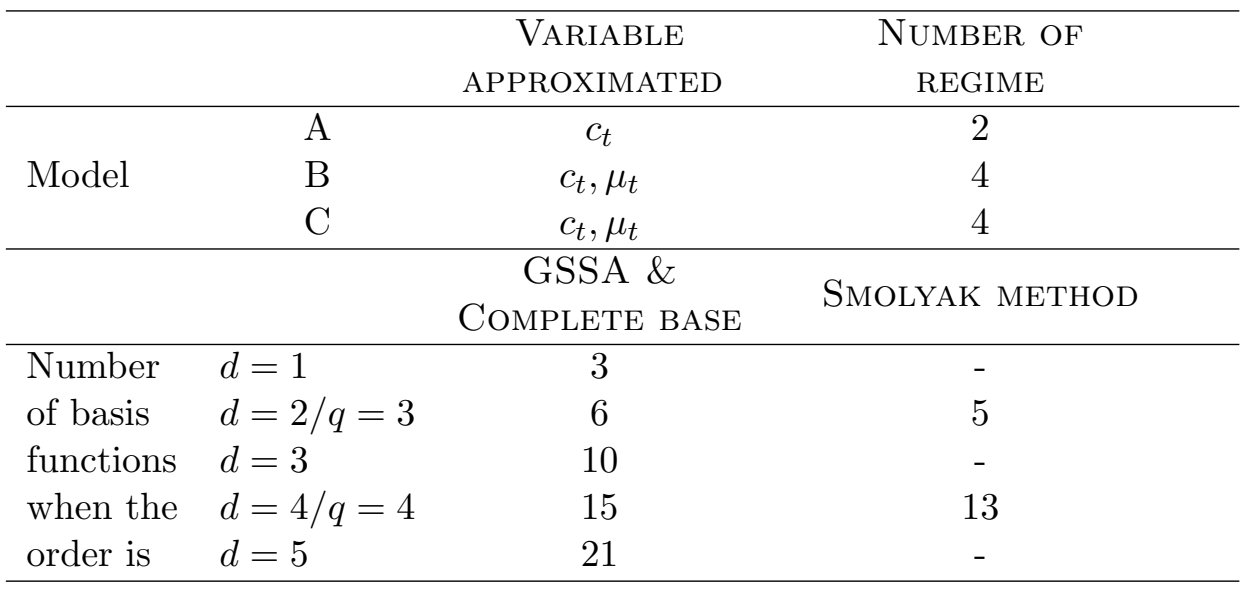

Table 4: Characteristics of the algorithms The number of regime is the theoretical one. It may differ from the number of regime that are effectively visited in equilibrium. For the Smolyak method, we report the value that are comparable to the complete basis i.e. the highest basis order is the same in the two cases. With order 2 in the complete base, $q=3$ and with order 4 in the complete base $q=4$.

\section{B.7 General algorithm - complete base}

We illustrate the algorithm with the benchmark model A (two government spending regime), 4-th order chebyshev polynomial and 10 Hermite quadrature nodes. The algorithm then consist in finding the coefficients $\Theta(1), \Theta(2)$. The Smolyak method involves the same steps as in the complete base except that the size of the grid and the number of polynomial basis are different.

\section{B.7.1 Algorithm}

Step 1 - Initialization Choose the order of the Chebyshev polynomial $\boldsymbol{D}$. Denote by $i$ the $i$-th iteration. At this stage, $i=0$. Set a convergence criteria $\underline{b}$. Initialize the coefficients $\Theta(1)^{i}, \Theta(2)^{i}$ using OLS regression on a stochastic simulation computed from the solution of a perturbation method ${ }^{11}$. We consider $\boldsymbol{D}=4, \boldsymbol{M}=\boldsymbol{D}+1=5$ and $\boldsymbol{K}=2$.

\footnotetext{
${ }^{11}$ This step is achieve thanks to the Dynare software. We consider a perturbation method of order 2 and assume that government expenditure are constant and equal to $g_{1}$.
} 
Step 2 - Multidimensional grid Compute the multidimensional grid $\mathcal{G}$ of the two continuous state variables, imposing the deterministic steady state to be equidistant from the upper bound and the lower bound of the grid in each dimension. $\mathcal{G}=\left[\mathcal{G}^{z} \mathcal{G}^{k}\right]$ is then a $[25 \times 2]$ matrix.

Step 3 - Today's regime Compute period t regime, given last period regime $n=1, \ldots, N$. Then for $j=1, \ldots, M^{K}$ and $n=1, \ldots, N$ :

a. Output: $y_{j}=\mathcal{G}_{j}^{k} \exp \left(\mathcal{G}_{j}^{z}\right)$

b. Regime: $s_{j n}=1 \times \mathbb{1}_{\left\{y_{j}>\underline{y}(n)\right\}}+2 \times \mathbb{1}_{\left\{y_{j} \leq \underline{y}(n)\right\}}$

Step 4 - Today's policy rules For $j=1, \ldots, M^{K}$ and $n=1, \ldots, N$ get:

a. Consumption: $c_{j n}=P\left(\mathcal{G}_{j}^{z}, \mathcal{G}_{j}^{k}, \Theta\left(s_{j n}\right)\right)$

b. Capital: $k_{j n}=y_{j}+(1-\delta) \mathcal{G}_{j}^{k}-c_{j n}-g\left(s_{j n}\right)$

Step 5 - Expectation Set Gauss-Hermite quadratures nodes $\boldsymbol{e}$ and weights $\omega$. We consider $H=10$ quadrature nodes. Denote by $v_{j n h}^{\prime}$ the $j$-th elements of the next period variable $v=z, k, s, y, c$ in regime $n$ at quadrature node $h$. The quadrature rule expends variables to account for potential future values of the shock. Then, for $n=1, \ldots, N, j=1, \ldots, J$ and $h=1, \ldots, H$ :

a. Compute next period variables:

$$
\begin{aligned}
z_{j n h}^{\prime} & =\rho_{z} \mathcal{G}_{j}^{z}+\sigma_{z} \boldsymbol{e}_{h} \sqrt{2} \\
y_{j n h}^{\prime} & =\exp \left(z_{j n h}^{\prime}\right) k_{j n}^{\alpha} \\
s_{j n h}^{\prime} & =1 \times \mathbb{1}_{\left\{y_{j n h}^{\prime}>\underline{y}(n)\right\}}+2 \times \mathbb{1}_{\left\{y_{j n h}^{\prime} \leq \underline{y}(n)\right\}} \\
c_{j n h}^{\prime} & =P\left(z_{j n h}^{\prime}, k_{j n}, \Theta\left(s_{j n h}^{\prime}\right)\right)
\end{aligned}
$$

b. Compute the expectation:

$$
\begin{aligned}
\mathcal{E}_{j n h} & =\beta\left(c_{j n h}^{\prime}\right)^{\sigma}\left(\exp \left(z_{j n h}^{\prime}\right) \alpha k_{j n}^{\alpha-1}+1-\delta\right) \\
\Xi_{j n} & =\frac{1}{\sqrt{\pi}} \sum_{h=1}^{H} \omega_{h} \mathcal{E}_{j n h}
\end{aligned}
$$

Step 6 - Estimate coefficients The new value of the coefficients is calculated using OLS. The dependent variables is pined down by the expectation terms and the explanatory variables is calculated from the basis function of the Chebyshev Polynomial. For $n=1, \ldots, N$ 
a. Pined down the estimate of today's consumption:

$$
\hat{c}_{j n}=\left(\Xi_{j n}^{i}\right)^{-\frac{1}{\sigma}}-\rho g\left(s_{j n}\right)
$$

b. Regress the estimate of consumption on the basis functions to get a new value for the coefficients:

$$
\Theta^{i+1}(n)=\left(\mathcal{B}^{C}(\mathcal{G})^{\top} \mathcal{B}^{C}(\mathcal{G})\right)^{-1}\left(\mathcal{B}^{C}(\mathcal{G})^{\top} \hat{c}_{n}\right)
$$

Step 7 - Euler residuals Compute the average Euler residuals in each regime $\left(c_{j n}\right.$ is defined in Step 4.a.):

$$
E E R_{n}^{i+1}=\log _{10}\left(\frac{1}{J} \sum_{j=1}^{J}\left|1-\frac{c_{j n}}{\hat{c}_{j n}}\right|\right)
$$

Step 8 - Criteria We consider two convergence criteria:

a. Policy rule convergence

$$
b_{n}=\frac{\left\|\Theta(n)^{i+1}-\Theta(n)^{i}\right\|}{\left\|\Theta(n)^{i}\right\|}
$$

b. EER convergence. Denote by $\tau$ the number of last iterations over which we calculate the mean of the EER. The criterion is defined as the difference between the mean of EER over the last $\tau$ iterations and the EER of iteration $i-\tau$.

$$
b_{n}= \begin{cases}\left|E E R_{n}^{i-\tau}-\frac{1}{\tau} \sum_{u=1}^{\tau} E E R_{n}^{i+1-u}\right| & \text { if } i<\tau \\ \underline{b} & \text { Otherwise }\end{cases}
$$

The final convergence criteria is

$$
b^{i}=\max \left(b_{1}, \ldots, b_{N}\right)
$$

\section{Step 9 - Convergence}

a. Check if $b^{i}<\underline{b}$. If it is the case, stop the algorithm. 
b. Otherwise, if $b^{i} \geq \underline{b}$, set $i=i+1$ and go back to Step 4 .

c. To achieve a convergence in the algorithm we use a smoothing parameter for the update of the policy rules:

$$
\Theta(n)^{i+1}=\gamma^{i} \Theta(n)^{i}+\left(1-\gamma^{i}\right) \Theta(n)^{i+1}
$$

where $\gamma^{i}$ depends on the iteration with the following values:

$$
\gamma^{i}= \begin{cases}\gamma_{1} & \text { if } i<i_{\min } \\ \gamma_{2} & \text { if } i_{\min } \leq i<i_{\max } \\ \gamma_{3} & \text { if } i \geq i_{\max }\end{cases}
$$

\section{B.7.2 Remarks}

- Step 1-3 are computed just once. The regime in period $t$ (Step 3) is based on capital and the productivity shock, both of them being given by the grid $\mathcal{G}$. If $s_{t}$ was function of $c_{t}$ it would have been necessary to recompute (Step 3) at each iteration $i$.

\section{B.8 GSSA}

In the GSSA approach, the major difference is the build of the endogenous grid. The function approximation is the same as in the complete base approach. We borrow from Maliar \& Maliar (2015) the EDS technique to select the representative points. The detailed step of the algorithm are as follow:

\section{B.8.1 Algorithm}

Step 1 - Initialization Choose the order of the Chebyshev polynomial $\boldsymbol{D}$. Denote by $i$ the $i$-th iteration. At this stage, $i=0$. Set a convergence criteria b. Initialize the coefficients $\Theta(1)^{i}, \Theta(2)^{i}$ using OLS regression on a stochastic simulation computed from the solution of a perturbation method ${ }^{12}$.

Step 2 - Stochastic simulations Compute a stochastic simulation over $T=20000$ periods using the same sequence of shock $\left\{\varepsilon_{t}^{z}\right\}_{t=0}^{T}$ as in Step 1 . Given initial condition of $k_{0}, z_{0}$ (deterministic steady state) and $s_{0}=1$, the steps for the stochastic simulation are as follow. For $t=1, \ldots T$ do:

$$
\text { a. Output: } y_{t}=k_{t-1} \exp \left(z_{t}\right)
$$

\footnotetext{
${ }^{12}$ This step is achieve thanks to the Dynare software. We consider a perturbation method of order 2 and assume that government expenditure are constant and equal to $g_{1}$.
} 
b. Regime: $s_{t}=1 \times \mathbb{1}_{\left\{y_{t}>\underline{y}\left(s_{t-1}\right)\right\}}+2 \times \mathbb{1}_{\left\{y_{t} \leq \underline{y}\left(s_{t-1}\right)\right\}}$

c. Consumption: $c_{t}=P\left(z_{t}, k_{t-1}, \Theta\left(s_{t}\right)\right)$

d. Capital: $k_{t}=y_{t}+(1-\delta) k_{t}-c_{t}-g\left(s_{t}\right)$

Step 3 - Representative points [Maliar \& Maliar (2015), section 2.2.2]. Set the parameter $\varepsilon_{n}, n=1, \ldots, N$. Given the time series previously calculated, define $X_{n}=\left\{z_{t}, k_{t-1} \mid s_{t}=n\right\}_{t=0}^{T}, n=1, \ldots, N$ as a set of points for which the regime is equal to $n$. Denote by $\mathcal{P}_{n}$ an empty set such that $\mathcal{P}_{n}=\{\varnothing\}$. For $n=1, \ldots, N$, do:

a. Select $x_{j} \in X_{n}$. Compute $\Delta\left(x_{j}, x_{\ell}\right), j \neq \ell$ to all $x_{j}$ in $X_{n}$ with the following formula

$$
\Delta\left(x_{j}, x_{\ell}\right)=\sqrt{\sum_{k=1}^{K}\left(P C_{j}^{k}-P C_{\ell}^{k}\right)^{2}}
$$

$\Delta\left(x_{j}, x_{\ell}\right)$ is the euclidian distance between principal components $(P C)$ of $X_{n}$ normalized to unit variance.

b. Eliminate from $X_{n}$ all $x_{j}$ for which $\Delta\left(x_{j}, x_{\ell}\right)<\varepsilon_{i}$

c. Add $x_{j}$ to $\mathcal{P}_{n}$ and eliminate it from $X_{n}$

d. Go back to step a. until $X_{n}=\{\varnothing\}$

Step 4 - Expectation Set Gauss-Hermite quadratures nodes $\boldsymbol{e}$ and weights $\omega$. We consider $H=10$ quadrature nodes. Denote by $x_{j n}^{z} \in \mathcal{P}_{n}$ and $x_{j n}^{k} \in \mathcal{P}_{n}$ the $j$-th elements (productivity shock and capital respectively) of the set $\mathcal{P}_{n}$ in regime $n$. Furthermore, denote by $v_{n j h}^{\prime}$ the $j$-th elements of the next period variable $v=z, k, s, y, c$ in regime $n$ at quadrature node $h$. The quadrature rule expends variables to account for potential future values of the shock. Then, for $n=1, \ldots, N, j=1, \ldots, J$ and $h=1, \ldots, H$ :

a. Compute next period variables:

$$
\begin{aligned}
z_{j n h}^{\prime} & =\rho_{z} x_{j n}^{z}+\sigma_{z} \boldsymbol{e}_{h} \sqrt{2} \\
y_{j n h}^{\prime} & =\exp \left(z_{j n h}^{\prime}\right)\left(x_{j n}^{k}\right)^{\alpha} \\
s_{j n h}^{\prime} & =1 \times \mathbb{1}_{\left\{y_{j n h}^{\prime}>\underline{y}(n)\right\}}+2 \times \mathbb{1}_{\left\{y_{j n h}^{\prime} \leq \underline{y}(n)\right\}} \\
c_{j n h}^{\prime} & =P\left(z_{n j h}^{\prime}, x_{j n}^{k}, \Theta\left(s_{n j h}^{\prime}\right)\right)
\end{aligned}
$$


b. Compute the expectation:

$$
\begin{aligned}
\mathcal{E}_{j n h} & =\beta\left(c_{j n h}^{\prime}\right)^{\sigma}\left(\exp \left(z_{j n h}^{\prime}\right) \alpha\left(x_{j n}^{k}\right)^{\alpha-1}+1-\delta\right) \\
\Xi_{j n} & =\frac{1}{\sqrt{\pi}} \sum_{h=1}^{H} \boldsymbol{\omega}_{h} \mathcal{E}_{j n h}
\end{aligned}
$$

Step 5 - Estimate coefficients The new value of the coefficients is calculated using OLS. The dependent variables is pined down by the expectation terms and the explanatory variables is calculated from the basis function of the Chebyshev Polynomial. For $n=1, \ldots, N$

a. Pined down the estimate of today's consumption:

$$
\hat{c}_{j n}=\Xi_{j n}^{-\frac{1}{\sigma}}-\rho g(n)
$$

$\boldsymbol{b}$. Regress the estimate of consumption on the basis functions to get a new value for the coefficients:

$$
\Theta(n)^{i+1}=\left(\mathcal{B}^{C}\left(\mathcal{P}_{n}\right)^{\top} \mathcal{B}^{C}\left(\mathcal{P}_{n}\right)\right)^{-1}\left(\mathcal{B}^{C}\left(\mathcal{P}_{n}\right)^{\top} \hat{c}_{n}\right)
$$

Step 6 - Euler residuals Compute the average Euler residuals in each regime:

$$
\begin{aligned}
E E R_{n}^{i+1} & =\log _{10}\left(\frac{1}{J} \sum_{j=1}^{J}\left|1-\frac{c_{j n}}{\hat{c}_{j n}}\right|\right) \\
c_{j n} & =P\left(x_{j n}^{z}, x_{j n}^{k}, \Theta(n)\right)
\end{aligned}
$$

Step 7 - Criteria We consider two convergence criteria:

a. Policy rule convergence

$$
b_{n}=\frac{\left\|\Theta(n)^{i+1}-\Theta(n)^{i}\right\|}{\left\|\Theta(n)^{i}\right\|}
$$


b. EER convergence. Denote by $\tau$ the number of last iterations over which we calculate the mean of the EER. We calculate the criterion by evaluating the stability of the EER over the last $\tau$ iterations.

$$
b_{n}= \begin{cases}\left|E E R_{n}^{i-\tau}-\frac{1}{\tau} \sum_{u=1}^{\tau} E E R_{n}^{i+1-u}\right| & \text { if } i<\tau \\ \underline{b} & \text { Otherwise }\end{cases}
$$

The final convergence criteria is

$$
b^{i}=\max \left(b_{1}, \ldots, b_{N}\right)
$$

\section{Step 8 - Convergence}

a. Check if $b^{i}<\underline{b}$. If it is the case, stop the algorithm.

b. Otherwise, if $b^{i} \geq \underline{b}$, set $i=i+1$ and go back to Step 2 .

c. To achieve a convergence in the algorithm we use a smoothing parameter for the update of the policy rules:

$$
\Theta(n)^{i+1}=\gamma^{i} \Theta(n)^{i}+\left(1-\gamma^{i}\right) \Theta(n)^{i+1}
$$

where $\gamma^{i}$ depends on the iteration with the following values:

$$
\gamma^{i}= \begin{cases}\gamma_{1} & \text { if } i<i_{\min } \\ \gamma_{2} & \text { if } i_{\min } \leq i<i_{\max } \\ \gamma_{3} & \text { if } i \geq i_{\max }\end{cases}
$$

\section{B.8.2 Remarks}

- While in most cases the algorithm converges without any need for perturbation methods for initializing the coefficients (Step 1), we found this strategy more efficient even if there are no RS when running the perturbation. An alternative could be the use of low order polynomial (e.g. order 2) to initialize the algorithm with higher order polynomials.

- Maliar \& Maliar (2015) raised an important question: How often do we need to reconstruct the EDS grid in a given application? The construction of the EDS grid is very fast in our model but can be computationally expensive in models with several state variables or if the model is solved several times e.g. for estimation. They suggest that the EDS grid can actually be computed just once. By "once" we understand 
that the set $\mathcal{P}_{n}$ has not the same values but the same elements from $X_{n}$. Said differently, the j-th element of the stochastic simulations are always picked whatever the value of the state variables ${ }^{13}$. Their idea is that the set of representative points remains representative from one iteration to another. In their example, they show that the EDS grid is able to autocorrect itself.

While this strategy works well in their setup, there is no guarantee that it works in every model. In our model characterized by RS some representative points of a regime $n$ may switch regime from one iteration to another. The policy rules can be either evaluated on a part of the state space that is never visited by regime $n$, or not evaluated on a part of the state space that would have been relevant to the decision rules if the EDS grid was recalculated. For this reason we recalculate the EDS grid at each iteration. An alternative could be the update of the EDS grid only every 5 or 10 iterations.

\section{B.9 Accuracy}

We borrow from Judd $(1992,1998)$ the accuracy test that consists in the evaluation of the residuals of the Euler equations calculated on a stochastic simulation. It examines if the policy functions are accurately approximated over two consecutive periods. The ideas is to compute the one-period optimization error in relation to current consumption. For the GSSA the Euler equation errors are evaluated on all simulated points, not just the point inside the EDS. We define the two hand-side of the Euler equation as (dropping time subscript):

$$
\begin{aligned}
c & =P(\boldsymbol{x} ; \Theta(s)) \\
\hat{c} & =\left[\beta E P\left(\boldsymbol{x}^{\prime} ; \Theta\left(s^{\prime}\right)\right)^{-\sigma}\left(\exp \left(z^{\prime}\right) \alpha k^{\prime \alpha-1}+1-\delta\right)\right]^{-\frac{1}{\sigma}}
\end{aligned}
$$

$\hat{c}$ is evaluated thanks to Monte Carlo integration techniques. Formally, the integral writes

\footnotetext{
${ }^{13}$ In Matlab, the EDS procedure gives the representative points of the state variables and the line numbers associated to the stochastic simulations. When EDS are computed once, the line numbers are held constant, not the value of the state variables.
} 


$$
\begin{aligned}
& {\left[\beta \int_{-\infty}^{\infty} P\left(k^{\prime}, z \rho+\varepsilon^{\prime} ; \Theta\left(\psi\left(s, \Gamma\left(\boldsymbol{x}, \varepsilon^{\prime} ; \Theta(s)\right)\right)\right)\right)^{-\sigma}\left(\exp \left(z \rho+\varepsilon^{\prime}\right) \alpha k^{\prime \alpha-1}+1-\delta\right) f\left(\varepsilon^{\prime}\right) d \varepsilon^{\prime}\right]^{-\frac{1}{\sigma}}} \\
& {\left[\beta \sum_{\varepsilon^{\prime}} P\left(k^{\prime}, z \rho+\varepsilon^{\prime} ; \Theta\left(\psi\left(s, \Gamma\left(\boldsymbol{x}, \varepsilon^{\prime} ; \Theta(s)\right)\right)\right)\right)^{-\sigma}\left(\exp \left(z \rho+\varepsilon^{\prime}\right) \alpha k^{\prime \alpha-1}+1-\delta\right) d \varepsilon^{\prime}\right]^{-\frac{1}{\sigma}}}
\end{aligned}
$$

For each period $t$ we draw 10000 value of $\varepsilon^{\prime}$ from a normal distribution $\mathcal{N}\left(0, \sigma_{z}^{2}\right)$. The Euler Equation Errors writes:

$$
E E E_{t}=\log _{10}\left(\frac{1}{T} \sum_{t=2}^{T}\left|1-\frac{c_{t}}{\hat{c}_{t}}\right|\right)
$$

\section{B.10 Convergence}

Convergence is controlled through the value of the parameter $\gamma^{i}$ and the iteration step by which the convergence parameter changes value. As described in the algorithm, we consider thee step which amount of setting $i_{\min }$ and $i_{\max }$.

Convergence is displayed in Figure 12 and the value of the convergence

\begin{tabular}{|c|c|c|c|c|c|c|c|c|c|}
\hline \multirow{2}{*}{ MODEL } & \multicolumn{3}{|c|}{ GSSA } & \multicolumn{3}{|c|}{ COMPlete BASE } & \multicolumn{3}{|c|}{ SMOLYAK METHOD } \\
\hline & $\gamma_{1}$ & $\gamma_{2}$ & $\gamma_{3}$ & $\gamma_{1}$ & $\gamma_{2}$ & $\gamma_{3}$ & $\gamma_{1}$ & $\gamma_{2}$ & $\gamma_{3}$ \\
\hline $\mathrm{A}$ & 0.1 & 0.5 & 1 & 0.05 & 0.5 & 0.7 & 0.05 & 0.5 & 0.8 \\
\hline B & 0.1 & 0.5 & 1 & 0.05 & 0.5 & 0.8 & 0.1 & 0.4 & 0.6 \\
\hline \multirow[t]{2}{*}{$\mathrm{C}$} & 0.1 & 0.5 & 1 & 0.05 & 0.5 & 0.8 & 0.1 & 0.4 & 0.6 \\
\hline & & $i_{\min }$ & $i_{\max }$ & & $i_{\min }$ & $i_{\max }$ & & $i_{\min }$ & $i_{\max }$ \\
\hline $\mathrm{A}$ & & 30 & 60 & & 100 & 200 & & 100 & 200 \\
\hline B & & 30 & 60 & & 100 & 200 & & 100 & 500 \\
\hline $\mathrm{C}$ & & 30 & 60 & & 100 & 200 & & 100 & 200 \\
\hline
\end{tabular}
parameter are given by table 5 .

Table 5: Convergence parameters 


\section{Figure 12: Convergence of the GSSA algorithm}

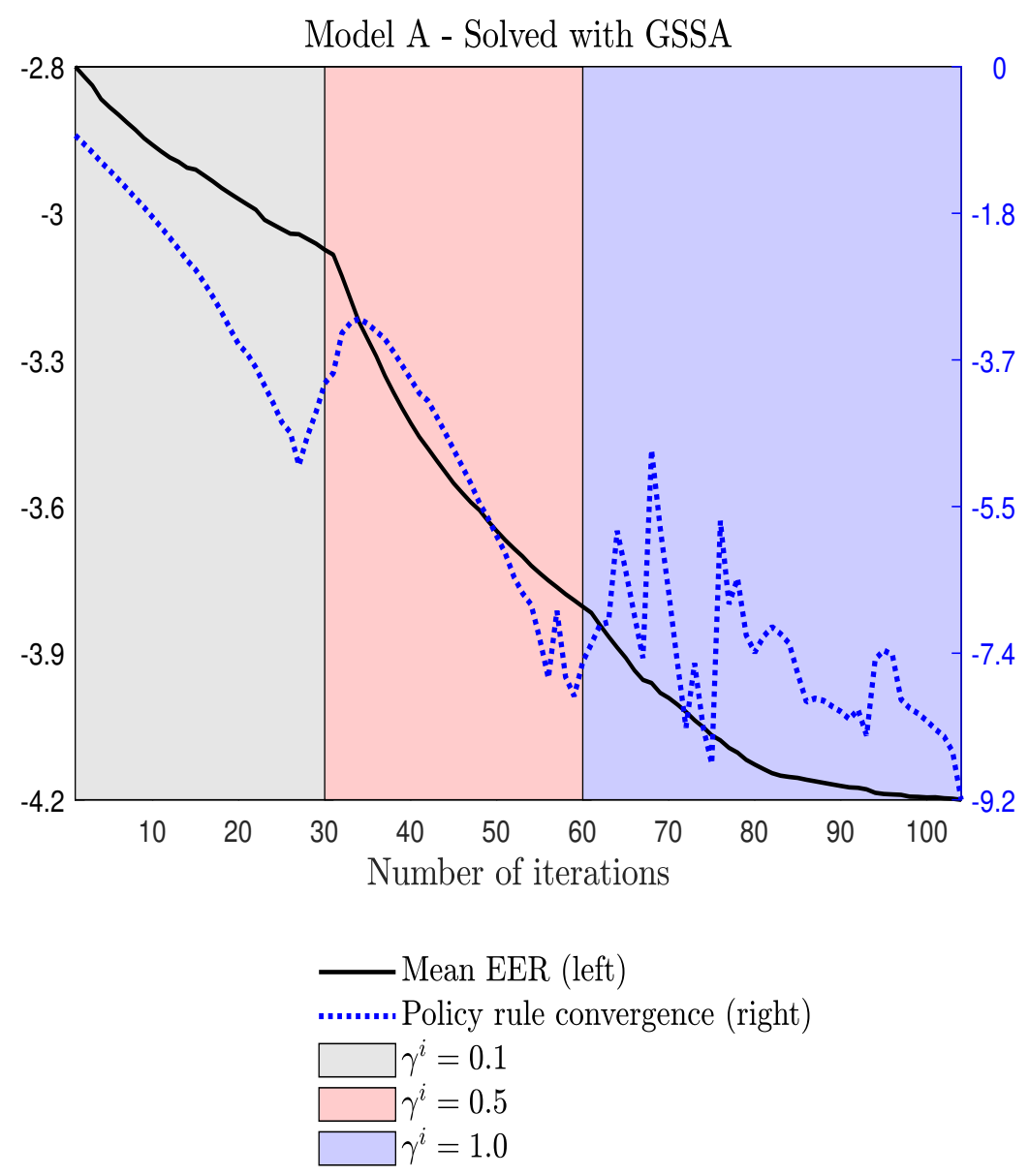

For the sake of exposition, we present $\log \left(b^{i}\right)$ instead of $b^{i}$ from Step 7 of the GSSA algorithm and Step $\boldsymbol{7}$ of the complete base approach. Left scale is the log10 basis. EER (black solid line) are the Euler equation errors $\left(L_{1}\right)$ calculated on the representative points for the GSSA. There are not calculated on the full stochastic simulations (contrary to the ones presented in Table 3 of the main paper).

- The GSSA algorithm converges in 150 iterations in the model A and $\mathrm{C}$ and it converges in 236 iterations in model B. When the smoothing parameter changes value at iteration 30 there is a bump in the convergence of the policy rule as well as in the EER stability. Except in Model $\mathrm{C}$, the EER declines monotonically with the number of iterations. 
- Over the three models, the computational time of the projection method is low but the convergence is not as stable as the GSSA. The algorithms require some fine tuning to ensure convergence. The number of iterations in the Smolyak method is several time higher than the GSSA for Model A and B.

\section{B.11 Hardware and parallelization}

- All the programs are run on Matlab R2017b. This last version is faster for loop of type for than previous versions. The computer used is a laptop with processor i9-8950HK (6 cores + hyper threading, 2.9Ghz on all core and $5 \mathrm{Ghz}$ in turbo mode on a single core), 32Gb RAM, GeForce GTX 1080max-Q and SSD Samsung 970 pro M.2 PCIe, Windows 10.

- Throughout the algorithms, no parallel computing are made for the sake of clarity. However, the GSSA can easily be parallelized either on the CPU or on the Graphical Processing Unit (GPU). See for instance Aldrich (2014) and Fernandez-Villaverde \& Valencia (2018). There are several way for using parallel computation ${ }^{14}$. We list two potential candidates that could reduce significantly the computational time in larger models.

1. It is easy to parallelize Step $\mathbf{2}$ by running multiple stochastic simulations together (with shorter horizon for each), gather them and go to Step 3.

2. An obvious trick is to evaluate in parallel the expectations (Step 4) for each regime since they can be computed separately. Indeed, the computation of the quadrature in one regime is made on a representative set of points that belongs to the regime. Consequently, we can use parallel computation over each regime. It is worth noting that in Step 4 we can also calculate in parallel each $\Xi_{j n}$ since we are dealing with representative points $j$ and we do not need to know $j$ to compute $\Xi_{\ell n}, j \neq \ell$.

\footnotetext{
${ }^{14}$ Since the best choice for TBRS model is the GSSA, we will only discuss parallel computing for GSSA.
} 


\title{
C Supplementary simulations
}

\section{C.1 EDS grid in models with irreversible investment}

\author{
Figure 13: EDS grid
}

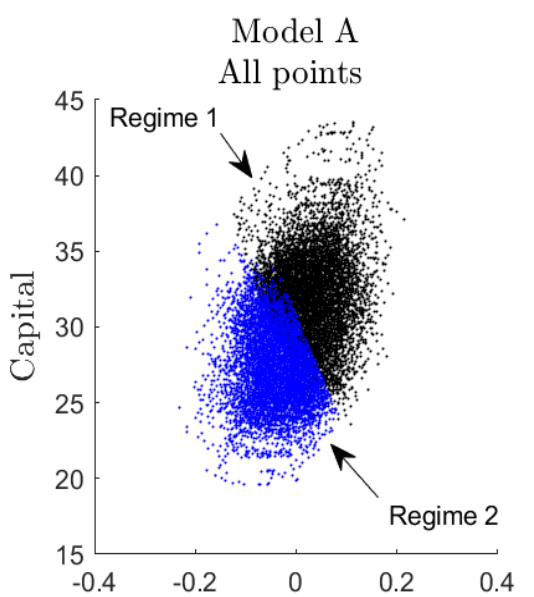

All points vs EDS
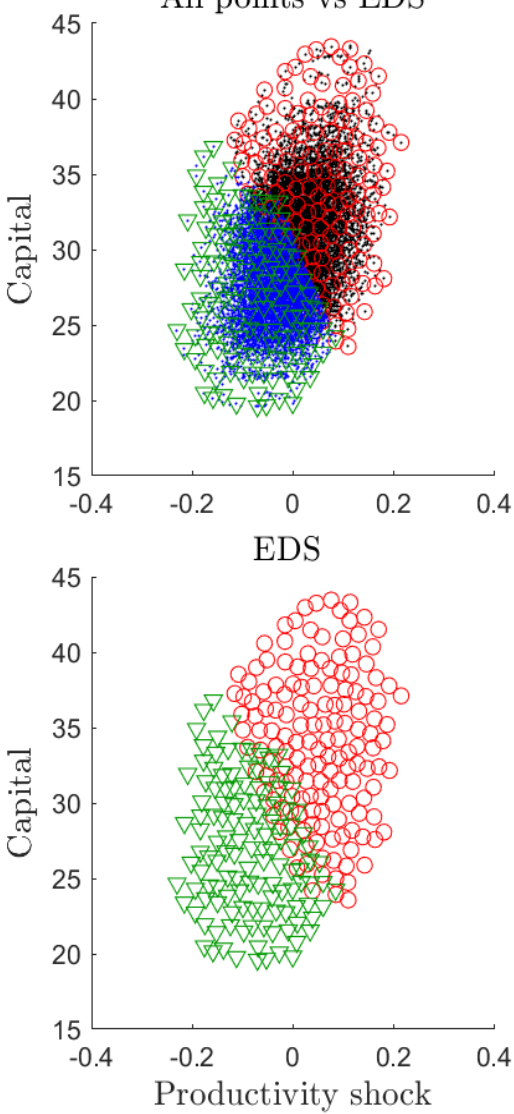

Model B

All points

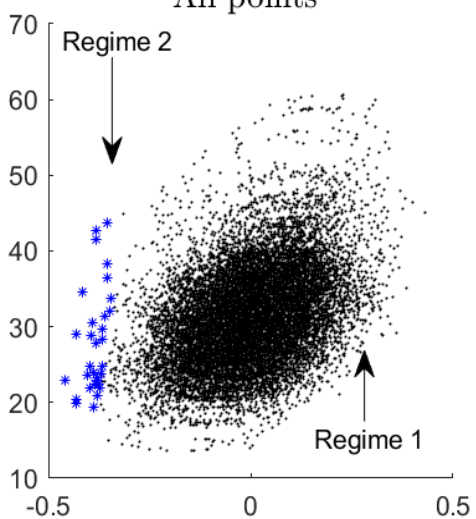

All points vs EDS

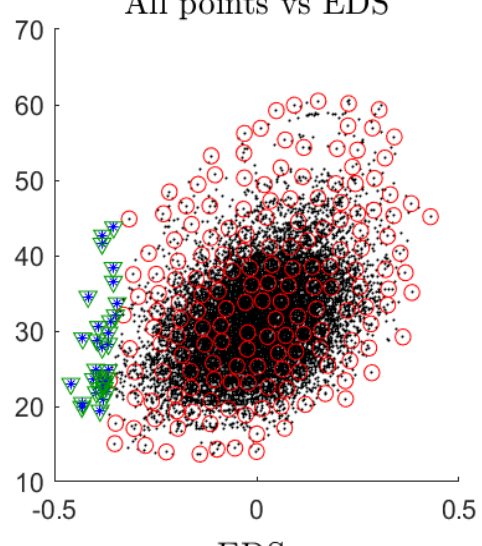

EDS

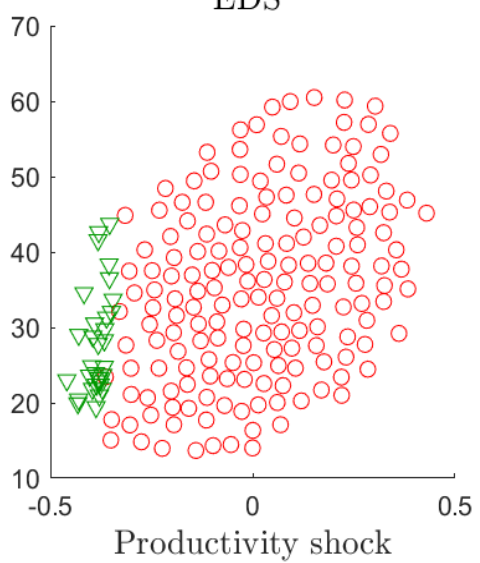

Model C

All points

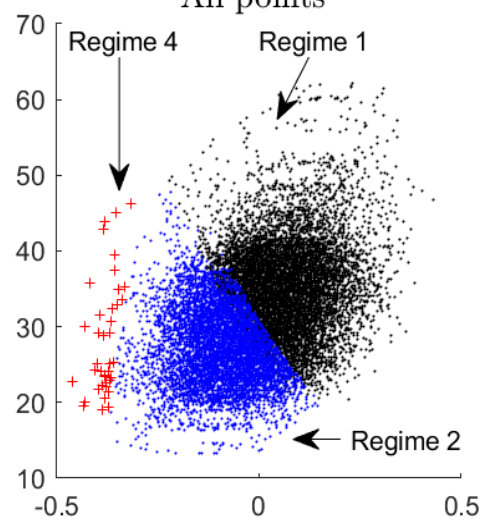

All points vs EDS

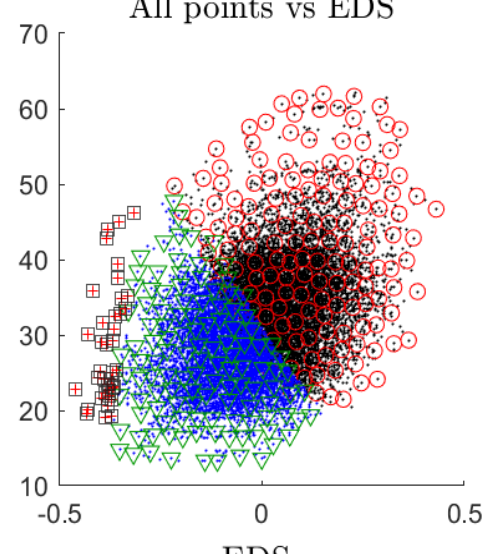

EDS

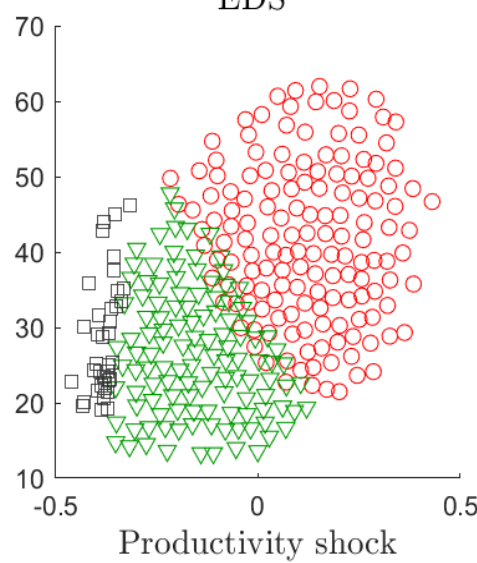

Grid points are computed on a stochastic simulation of 20000 observations under the three different models. Simulated points from the stochastic methods are the ones from the last iteration. Chebyshev polynomials are of order 4. Model A: benchmark model, two asymmetric regimes based on government spending. Model B: Irreversible investment, two symmetric regimes based on the investment constraint. Model C: Benchmark + Irreversible investment, four potential regimes 


\section{C.2 Model with uncertain RS rule}

\section{Figure 14: Euler Equation Errors}

- $s_{t}=1 \cdot s_{t}=2 @ \mathrm{KS}$ density $s_{t}=1 @ \mathrm{KS}$ density $s_{t}=2$

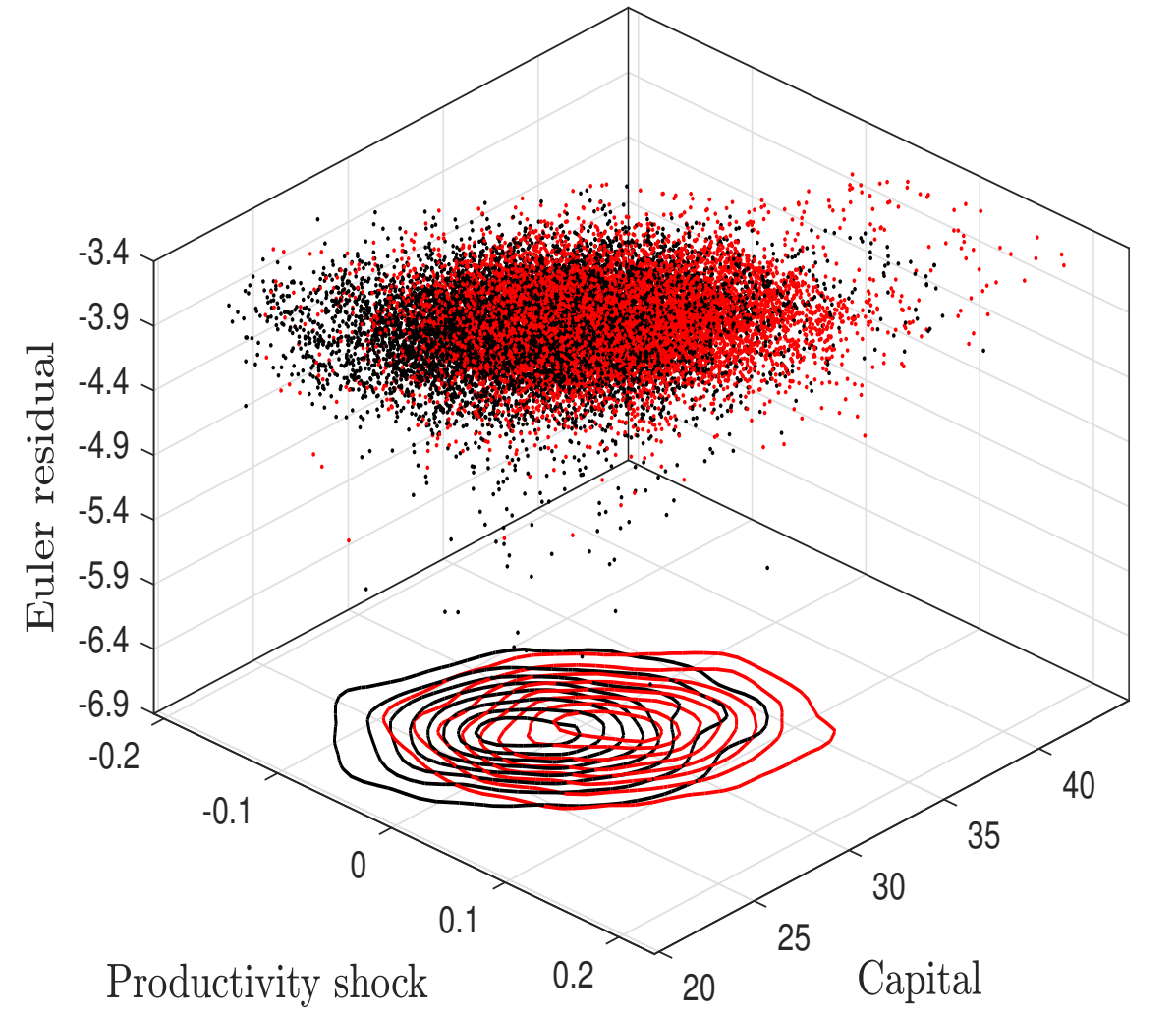

Euler equation errors (in log10 units) are computed on a stochastic simulation with 20000 draws from $\varepsilon_{t}^{z}$. Bivariate smooth Kernel density estimate are displayed for each regime.

\section{References}

Aldrich, E. M. (2014). Chapter 10 - GPU Computing in Economics. In K. Schmedders \& K. L. Judd (Eds.), Handbook of Computational Economics, volume 3 of Handbook of Computational Economics Vol. 3 (pp. 557-598). Elsevier.

Barthelmann, V., Novak, E., \& Ritter, K. (2000). High dimensional polynomial interpolation on sparse grids. Advances in Computational Mathematics, 12(4), 273-288. 
Barthélemy, J. \& Marx, M. (2017). Solving endogenous regime switching models. Journal of Economic Dynamics and Control, 77, 1-25.

Barthélemy, J. \& Marx, M. (2019). Monetary policy switching and indeterminacy. Quantitative Economics, 10(1), 353-385. Number: 1.

Benigno, G., Foerster, A., Otrok, C., \& Rebucci, A. (2020). Estimating Macroeconomic Models of Financial Crises: An Endogenous RegimeSwitching Approach. Technical Report 26935, National Bureau of Economic Research, Inc.

Bi, H., Leeper, E. M., \& Leith, C. (2013). Uncertain Fiscal Consolidations. The Economic Journal, 123(566), F31-F63.

Bianchi, F. (2013). Regime Switches, Agents' Beliefs, and Post-World War II U.S. Macroeconomic Dynamics. The Review of Economic Studies, 80(2), 463-490. Publisher: Oxford Academic.

Binning, A. \& Maih, J. (2017). Modelling Occasionally Binding Constraints Using Regime-Switching. Technical Report 2017/23, Norges Bank.

Boneva, L., Harrison, R., \& Waldron, M. (2018). Threshold-based forward guidance. Journal of Economic Dynamics and Control, 90, 138-155.

Brumm, J. \& Scheidegger, S. (2017). Using Adaptive Sparse Grids to Solve High-Dimensional Dynamic Models. Econometrica, 85(5), 1575-1612.

Canova, F., Ferroni, F., \& Matthes, C. (2020). Detecting and Analyzing the Effects of Time-Varying Parameters in Dsge Models. International Economic Review, 61(1), 105-125.

Chang, Y., Choi, Y., \& Park, J. Y. (2017). A new approach to model regime switching. Journal of Econometrics, 196(1), 127 - 143.

Chang, Y., Maih, J., \& Tan, F. (2019). Origins of monetary policy shifts: A new approach to regime switching in dsge models. Norges Bank Working Paper.

Christiano, L. J. \& Fisher, J. D. M. (2000). Algorithms for solving dynamic models with occasionally binding constraints. Journal of Economic Dynamics and Control, 24(8), 1179-1232.

Chung, H., Davig, T., \& Leeper, E. M. (2007). Monetary and Fiscal Policy Switching. Journal of Money, Credit and Banking, 39(4), 809-842. _eprint: https://onlinelibrary.wiley.com/doi/pdf/10.1111/j.15384616.2007.00047.x. 
Davig, T., Leeper, E. M., Clarida, R. H., \& Lindé, J. (2006). Endogenous monetary policy regime change [with comments]. NBER International Seminar on Macroeconomics, (pp. 345-391).

Demkowicz, L. (2006). Computing with hp-ADAPTIVE FINITE ELEMENTS: Volume 1 One and Two Dimensional Elliptic and Maxwell Problems. CRC Press.

Demkowicz, L., Rachowicz, W., \& Devloo, P. (2002). A Fully Automatic hp -Adaptivity. Journal of Scientific Computing, 17(1), 117-142.

Farmer, R. E. A., Waggoner, D. F., \& Zha, T. (2009). Understanding Markov-switching rational expectations models. Journal of Economic Theory, 144(5), 1849-1867.

Fernandez-Villaverde, J., Rubio-Ramirez, J. F., \& Schorfheide, F. (2016). Solution and Estimation Methods for DSGE Models. In Handbook of Macroeconomics, volume 2 (pp. 527-724). Elsevier.

Fernandez-Villaverde, J. \& Valencia, D. Z. (2018). A Practical Guide to Parallelization in Economics. Technical Report 24561, National Bureau of Economic Research, Inc.

Fève, P., Matheron, J., \& Sahuc, J.-G. (2013). A Pitfall with Estimated DSGE-Based Government Spending Multipliers. American Economic Journal: Macroeconomics, 5(4), 141-178.

Halton, J. H. (1964). Algorithm 247: Radical-inverse quasi-random point sequence.

Hamilton, J. D. (1994). Time Series Analysis. Princeton, N.J: Princeton University Press.

Hamilton, J. D. (2016). Chapter 3 - Macroeconomic Regimes and Regime Shifts. In J. B. Taylor \& H. Uhlig (Eds.), Handbook of Macroeconomics, volume 2 (pp. 163-201). Elsevier.

Hirose, Y. \& Sunakawa, T. (2019). Review of solution and estimation methods for nonlinear dynamic stochastic general equilibrium models with the zero lower bound. The Japanese Economic Review, 70(1), 51-104.

Judd, K. L. (1992). Projection methods for solving aggregate growth models. Journal of Economic Theory, 58(2), 410-452. 
Judd, K. L. (1998). Numerical Methods in Economics, volume 1. The MIT Press.

Judd, K. L., Maliar, L., \& Maliar, S. (2011). Numerically stable and accurate stochastic simulation approaches for solving dynamic economic models. Quantitative Economics, 2(2), 173-210.

Judd, K. L., Maliar, L., Maliar, S., \& Valero, R. (2014). Smolyak method for solving dynamic economic models: Lagrange interpolation, anisotropic grid and adaptive domain. Journal of Economic Dynamics and Control, $44,92-123$.

Krueger, D. \& Kubler, F. (2004). Computing equilibrium in OLG models with stochastic production. Journal of Economic Dynamics and Control, 28(7), 1411-1436.

Leeper, E. M. \& Zha, T. (2003). Modest policy interventions. Journal of Monetary Economics, 50(8), 1673-1700.

Liu, Z., Waggoner, D. F., \& Zha, T. (2011). Sources of macroeconomic fluctuations: A regime-switching DSGE approach. Quantitative Economics, 2(2), 251-301. _eprint: https://onlinelibrary.wiley.com/doi/pdf/10.3982/QE71.

Maliar, L. \& Maliar, S. (2015). Merging simulation and projection approaches to solve high dimensional problems with an application to a new Keynesian model. Quantitative Economics, 6(1), 1-47.

Malin, B. A., Krueger, D., \& Kubler, F. (2011). Solving the multi-country real business cycle model using a Smolyak-collocation method. Journal of Economic Dynamics and Control, 35(2), 229-239.

Nakata, T. (2017). Uncertainty at the Zero Lower Bound. American Economic Journal: Macroeconomics, 9(3), 186-221.

Patera, A. T. (1984). A spectral element method for fluid dynamics: Laminar flow in a channel expansion. Journal of Computational Physics, 54(3), 468488.

Sims, C. A. \& Zha, T. (2006). Were There Regime Switches in U.S. Monetary Policy? American Economic Review, 96(1), 54-81.

Smolyak, S. (1963). Quadrature and interpolation formulas for tensor products of certain classes of functions. 
Svensson, L. E. O. \& Williams, N. (2009). Optimal Monetary Policy under Uncertainty in DSGE Models: A Markov Jump-Linear-Quadratic Approach. In Central Banking, Analysis, and Economic Policies Book Series, volume 13 (pp. 077-114). Central Bank of Chile. 